$$
\text { Mevised Version }
$$

SLOSHING DYNAMICS MODULATED FLUID ANGULAR MOMENTUM

AND MOMENT FLUCTUATIONS DRIVEN BY ORBITAL GRAVITY

GRADIENT AND JITTER ACCELERATIONS IN MICROGRAVITY

R. J. Hung and H. L. Pan

The University of Alabama in Huntsville

Huntsville, Alabama 35899 USA

(NASA-TM-111064) SLOSHING DYNAMICS

MODULATED FLUID ANGULAR MOMENTUM

N96-11215

ANO MOMENT FLUCTUATIONS DRIVEN BY

ORBITAL GRAVITY GRADIENT AND JITTER

ACCELERATIONS IN MICROGRAVITY

(Alabama Univ.) $42 \mathrm{p}$

$63 / 34 \quad 0065146$ 


\section{At stract}

The dynamical behavior of spacecraft propellant affected by the asymmetric combined gravity gradient and $j$ itter accelerations, in particular the effect of surface tension on partially-filled cotating flulds applicable to a full-scale Gravity Probe-B Spacecraft dewar tank has been investigated. Three different cases of orbital accelerations: (a) gravity gradient-dominated, (b) equally weighted between gravity gradient and jitter, and (c) gravity jitter-dominated accelerations are studied. The results of slosh wave excitation along the liquid-vapor interface induced by gravity gradient-dominated accelerations provide a torsional moment with tidal motion of bubble oscillations in the rotating dewar. The results are clearly seen from the twisting shape of the bubble oscillations driven by gravity gradient-dominated acceleration. The results of slosh wave excitation along the liquid-vapor interface induced by gravity jitter-dominated acceleration indicate the results of bubble motion in a manner of down-and-up and leftward-and-rightward movement of oscillation when the bubble is rotating with respect to rotating dewar axis. Fluctuations of angular momentum, fluid moment and bubble mass center caused by slosh wave excitations driven by gravity gradient acceleration or gravity jitter acceleration are also investigated. 


\section{Introduction}

In space experiments, superconducting sensors are used for gyro read-out and maintain a very low temperature for mechanical stability. The approaches to both cooling and control involve the use of superfluid liquid helium. For example, to test Einstein's General Relativity, the Gravity Probe-B (GP-B) Spacecraft adopts the boil-off from the cryogenic liquid helium dewar as a propellant to maintain the attitude control and drag-free operation of the spacecraft ${ }^{1}$. The potential problems for cryogenic liquid in the dewar container could be due to asymmetry in the static liquid helium distribution which is controlled by the capillary effect governed 1iquid-vapor interface disturbances in a microgravity environment.

In the absence of temperature gradient along the liquid-vapor interface for superfluid helium which drives Marangoni convection, the equilibrium shape of the interface in a rotating container is governed by a balance of capillary, centrifugal and gravitational forces in microgravity. The determination of liquid-vapor interface profiles based on computational experiments can uncover details of the capillary effect driven flow which can not be easily visualized or measured experimentally in a microgravity environment.

The instability of the capillary effect governed liquid-vapor interface profiles can be induced by the presence of longitudinal and lateral accelerations, vehicle vibration, and rotational fields of the spacecraft. Thus, slosh waves are excited, producing high and low frequency oscillations in the fluid systems. The sources of the residual accelerations range from the effects of the Earth's gravity gradient acceleration and jitter acceleration, which includes atmospheric drag on the spacecraft, spacecraft attitude motions arising from machinery vibrations, thruster firings, crew motion, etc. A recent study ${ }^{2}$ 
suggests that the high frequency accelerations may be unimportant in comparison to the residual motions caused by low frequency accelerations.

The time-dependent dynamical behavior of the capillary effect governed liquid-vapor-solid interface disturbances for rotating fluids in reduced gravity environments was simulated by numerically solving the Navier Stokes equations subject to the initial and boundary conditions ${ }^{3-4}$. At the interface between the liquid and the vapor fluids, both the kinematic surface boundary condition and the interface stress conditions for components tangential and normal to the interface were applied ${ }^{3-6}$. The initial conditions were adopted from the steady-state formulations developed by Hung et $a 1^{3}$ with an example in the geometry of the GP-B Spacecraft ${ }^{1}$. Some of the steady-state formulations of interface shapes were compared with the available experiments carried out by Leslie ${ }^{7}$ in a free-falling aircraft (KC-135). The experiments carried out by Mason et $a 1^{8}$ showed that the classical fluid mechanics theory is applicable for cryogenic liquid helium in large containers.

As for the effect of centrifugal force on the liquid helium and helium vapor with an example of the GP-B Spacecraft, a spin rate of up to about $1 \mathrm{rpm}$ will be imposed for instrument calibration in the early stages of the experiment. After calibration, the rotation rate will be reduced to its operational value of approximately $0.1 \mathrm{rpm}$.

As the spacecraft moves along the orbit, any fluid mass capable of motion relative to the spacecraft is subject to the acceleration that arises from the gravity gradients of the Earth ${ }^{9-11}$ and orbital environmental gravity jitter acceleration. In this paper, in addition to the ubiquitously available study of sloshing dynamics modulated liquid-vapor interface oscillations in normal and microgravity environment, we are particularly interested in the investigation of 
orbital acceleration, the combined gravity gradient and jitter accelerations, induced sloshing dynamics and the characteristics of its impact on orbital spacecraft fluid systems. In this study, comparisons are made of the dynamical evolution of sloshing dynamics excited moment and angular momentum fluctuations of fluid system for the combined gravity gradient and jitter accelerations at different background of gravity jitter accelerations due to the various ranges of environmental drag. In other words, the study is based on the orbital gravity gradient acceleration with a combination of three ranges of gravity jitter accelerations $\left(10^{-6}, 10^{-7}\right.$ and $\left.10^{-8} \mathrm{~g}_{0}\right)$ for the purpose of studying the dynamics of capillary effect governed liquid-vapor-solid interface disturbances and their effects on fluid moment and angular momentum disturbances.

II. Mathematical Model of Capillary Effect Governed Slosh Wave Excitation

\section{Along the Liquid-Vapor Interface}

Consider a closed circular dewar cylinder of an inner radius $a_{1}$, and an outer radius $a_{2}$, with height $L$, which is partially filled with cryogenic liquid helium, and rest of the ullage is filled with a helium vapor. Density and viscosity of liquid helium and helium vapor are $\rho_{L}, \mu_{L}, \rho_{v}$, and $\mu_{v}$, respectively. Let us use cylindrical coordinates ( $r, \theta, z)$, with corresponding velocity components $(u, v, w)$, and corresponding residual gravity acceleration, such as gravity gradient components $\left(a_{g g, r}, a_{B g, \theta}, a_{g g, z}\right)$ and gravity jitter components $\left(a_{g j, x}, a_{8 j, \theta}, a_{8 j, z}\right)$. The whole fluid system is spinning in the $z-a x i s$ with an angular velocity of $\omega$ and an angular acceleration of $\alpha$. The governing equations for non-inertia frame bound coordinates are illustrated in our recent studies ${ }^{12,13}$ and will not be repeated in this paper. In these formulations, $2 \rho \omega u$ and $2 \rho \omega \mathrm{V}$ denote Coriolis forces; $\rho$ ra, the angular acceleration; and $\rho r \omega^{2}$, the centrifugal force of spinning motion. 
In order to solve sloshing dynamics problems of liquid propellant systems In orbital spacecraft under a microgravity environment, one has to solve the governing non-inertia frame equations ${ }^{12,13}$ accompanied by a set of initial and boundary conditions. A detailed illustration of these initial and boundary conditions concerning the sloshing dynamics of fluid systems in microgravity were precisely given in our recent studies ${ }^{13.14}$ and will not be repeated in this paper. In this study, in order to show a realistic example, a full scale GP-B spacecraft propellant dewar tank with an inner radius of $12 \mathrm{~cm}$ and an outer radius of $68 \mathrm{~cm}$ and a height of $145 \mathrm{~cm}$ has been used in the numerical simulation. The propellant tank is 80 filled with cryogenic liquid helium and the rest of ullage is filled with helium vapor. The temperature of cryogenic helium is $1.8 \mathrm{~K}$. In this study the following data was used: 1iquid helium density $=146 \mathrm{~kg} / \mathrm{m}^{3}$, helium vapor density $=1.47 \mathrm{~kg} / \mathrm{m}^{3}$, fluid pressure $=1.66 \mathrm{~Pa}$, surface tension coefficient at the interface between liquid helium and helium vapor $=0.0353 \mathrm{~N} / \mathrm{m}$, liquid helium viscosity coefficient $=9.61 \times 10^{-9} \mathrm{~m}^{2} / \mathrm{s} ;$ and contact angle $=5^{\circ}$. The initial profiles of the liquid-vapor interface used in this study have been given explicitly through the steady state computations made by Hung and Leslie ${ }^{15}$ and Hung et al. 3,4,16, which were checked by experiments carried out by Leslife?.

A staggered grid for the velocity components is used in this computer program. The marker-and-cell method of studying fluid flows along a free surface is adopted ${ }^{17-20}$. The formulation for this method is valid for any arbitrary interface location between the grid points and is not limited to middle point interfaces ${ }^{19}$. An algorithm for a semi-implicit method ${ }^{20}$ was used as the procedure for modeling the flow field. The time step is determined automatically based on the size of the grid points and the velocity of flow fields. More than 200 times of iteration have been applied for each time step to assure the convergence of 
series. As for the volume conservation of the liquid, a deviation of less than 18 error of volume is always guaranteed before a move to the next time step. III. Dynamical Behavior of Liquid-Vapor Interface Disturbances Driven by Orbital Accelerations in Microgravity

For the case of the GP-B Spacecraft, which is an Earth satellite orbiting at $650 \mathrm{~km}$ altitude directly over the poles, the orbit period, $\tau_{0}$ can be computed from the following expression:

$$
\tau_{0}=2 \pi \frac{R_{c}^{3 / 2}}{R_{E} G_{0}^{1 / 2}}
$$

where $R_{E}$ denotes radius of Earth $(-6373 \mathrm{~km}) ; R_{c}$, radius of circular orbit (- $R_{E}$ $+h=7023 \mathrm{~km}) ; \mathrm{h}$, orbit altitude $(-650 \mathrm{~km})$; and $\mathrm{g}_{0}$, Earth gravity acceleration $\left(-9.81 \mathrm{~m} / \mathrm{s}^{2}\right)$. For the case of GP-B, the orbit period $\tau_{0}-97.6 \mathrm{~min}$, and orbit rate $\mathrm{n}=2 \pi / \tau_{0}-1.07 \times 10^{-3} \mathrm{rad} / \mathrm{s}$.

The gravity gradient acceleration acting on the fluid mass of spacecraft can be shown as

$$
\hat{a}_{g g}=-n^{2}\left[3\left(\hat{r}_{c} \cdot \hat{d}\right) \hat{r}_{c}-\hat{d}\right]
$$

where $\hat{a}_{\mathbf{g}}$ denotes gravity gradient acceleration vector; $\hat{a}$, vector from the fluid element to the spacecraft mass center; $\hat{r}_{c}$, unit vector from the spacecraft mass center to the center of the Earth; and $n$, the orbit rate.

For the case of the GP-B Spacecraft, it is assumed that the gravity exerted on the mass center of the spacecraft orbiting around the Earth on its specified orbit is zero. In other words, all the gravity acceleration exerted on the spacecraft is nothing but the gravity gradient acceleration which is defined in Equation (2). In this study, we are interested in investigating how combined gravity gradient and jitter accelerations affect the dynamical behavior of 
cryogenic fluid elements of helium.

For the convenience of mathematical calculation, let us describe all the parameters involved in Equation (2) in terms of cartesian coordinates. In order to match with the computer simulation, mathematical derivation are considered in the first quadrant. Figure 1 illustrates the geometrical relationship of the parameters shown in Equation (2).

Let us consider the fluid element of interest, $m$, located at $(r, \theta, z)$ in cylindrical coordinates and at $(x, y, z)$ in cartesian coordinates. As $|\hat{d}|$ is much smaller than the distance between the location of the GP-B Spacecraft geometric center to the center of the Earth, $\hat{\mathbf{r}}_{c}$ through the GP-B geometric center and $\dot{r}_{c}$ through the fluid element, $m$, is basically the same.

The gravity gradient acceleration acted on the fluid element located at ( $r$, $\theta, z)$, in non-inertia frame coordinates is shown as follows ${ }^{12,13,16}$ :

$$
\begin{gathered}
\hat{a}_{g g}=\left[\begin{array}{l}
a_{g g, r} \\
a_{g g, \theta} \\
a_{g g, z}
\end{array}\right]=-n^{2}\left[\begin{array}{ccc}
\cos (\theta+\omega t) & \sin (\theta+\omega t) & 0 \\
-\sin (\theta+\omega t) & \cos (\theta+\omega t) & 0 \\
0 & 0 & 1
\end{array}\right] . \\
\cdot\left[\begin{array}{c}
3\left[(z-L / 2) \cos \psi_{E}-r \cos (\theta+\omega t) \cdot \sin \psi_{E}\right] \sin \psi_{E}+r \cos (\theta+\omega t) \\
r \sin (\theta+\omega t) \\
-3\left[(z-L / 2) \cos \psi_{E}-r \cos (\theta+\omega t) \cdot \sin \Psi_{E}\right] \cos \Psi_{E}+(z-L / 2)
\end{array}\right]
\end{gathered}
$$

where $\psi_{\bar{E}}$ denotes the azimuth angle of Earth toward the location of spacecraft geometric center, or the angle between the spacecraft rotating axis and the radial direction from spacecraft geometric center to center of Earth; $n$, the orbit rate; and $t$, the time of spacecraft orbiting around the Earth.

At time $t=0$, the rotating axis of the spacecraft is aligned with the radial direction of Earth center to the spacecraft geometric center. An example is given by assuming that the spacecraft axis of rotation is linearly turning around $0^{\circ}$ to $360^{\circ}$ in $1200 \mathrm{~s}$, which is twice the period of spacecraft rotating 
time ( $0.1 \mathrm{rpm}$ is equivalent to a $600 \mathrm{~s}$ rotating period), when the spacecraft is orbiting around the Earth. Thus, $\psi_{E}$ can be defined as

$$
\Psi_{B}=2 \frac{\pi}{\tau} t
$$

where $T$ is the spacecraft gravity turn-around time (- $1200 \mathrm{~s}$ in this case); and $t$ is the time measured from the instant when the direction of the spacecraft rotating axis is aligned with the radial direction of the spacecraft mass center to the center of the Earth.

The fluctuations of residual gravity due to orbital environmental gravity jitter acceleration are modeled by the following equation:

$$
g=g_{B}\left[1+\frac{1}{2} \sin (2 \pi f t)\right]
$$

where $g_{B}$ denotes the orbital background gravity environment, and $f(\mathrm{~Hz})$ stands for the frequency of gravity-jitters.

In this study, three ranges of gravity jitter background with gravity of $10^{-6}, 10^{-7}$ and $10^{-8} \mathrm{go}$ due to different intensity of spacecraft atmospheric drag and rotating speed of $0.1 \mathrm{rpm}$ for normal GP-B spacecraft operation, and three frequencies of gravity jitter with the values of $0.1,1.0$ and $10 \mathrm{~Hz}$ have been considered for the investigation of the oscillations of the liquid-vapor interface. The components of gravity jitter acceleration in the non-inertia coordinate system shall be given by

$$
\hat{a}_{g j}=\left(a_{g j, I}, a_{g j, \theta}, a_{g j, z}\right)=\left[g \sin \psi_{E} \cos (\theta+\omega t),-g \sin \psi_{E} \sin (\theta+\omega t),-g \cos \Psi_{E}\right]
$$

The characteristics of gravity gradient and jitter accelerations, shown in Equations (3) and (6), respectively, are quite different. Gravity gradient acceleration shows the following characteristics: (a) acceleration acts on any 
fluid mass inside the container which increases two units of acceleration per unit of distance measured from the container mass center (point $o_{8}$ at Figure 1 ) to the location of the fluid mass parallel along the radial axis from the container mass center to the center of the Earth (parallel to unit vector $\dot{r}_{c}$ shown in Figure 1); (b) the acceleration acts on the fluid mass which decreases one unit of acceleration per unit of the shortest distance measured from the location of the fluid mass to the radial axis along the vector from the container mass center to the center of the Earth ${ }^{10}$. For example, Figure 2 shows the time variation of gravity gradient accelerations for a turn-around period of $1200 \mathrm{~s}$ with a container rotating speed of $0.1 \mathrm{rpm}$ for components along ( $x, y, z)$ directions acted on the fluid mass located at $(r, \theta, z)-(40 \mathrm{~cm}, \pi / 4,10 \mathrm{~cm})$. As the magnitude and direction of gravity gradient acceleration acted on each fluid mass is strongly dependent upon how far the location of the fluid mass deviates from the container mass center measured along the axis parallel to the vector $\hat{r}_{c}$ which varies with respect to time, it can be anticipated that the gravity gradient acceleration acted on the fluid mass is different for fluid mass at different locations in the container. Figure 2 shows that the magnitude of gravity gradient acceleration is on the order of $10^{-7} \mathrm{go}$. On the contrary to gravity gradient acceleration which acts different acceleration on the fluid mass at different locations in the container, gravity jitter acceleration drives the same acceleration on the fluid mass at different locations in the container. Figure 3 shows the time variation of gravity jitter accelerations for a turnaround period of $1200 \mathrm{~s}$ with a container rotating speed of $0.1 \mathrm{rpm}$ and a jitter frequency of $0.1 \mathrm{~Hz}$ for components along ( $x, y, z)$ directions acted on the fluid mass everywhere in the container. It is shown in Figure 2 that gravity gradient accelerations are on the order of $10^{-7}$ go for the spacecraft orbit chosen in 
this example, three ranges of background gravity, $10^{-6}, 10^{-7}$ and $10^{-8}$ go for gravity jitter accelerations correspond to the magnitude of acceleration higher, equal, and lower, respectively, than that of the gravity gradient acceleration acted on the fluid system of the spacecraft.

The equilibrium shape of the liquid-vapor interface for a rotating dewar under a residual gravity environment below $10^{-6} \mathrm{~g}$ and rotating speed of $0.1 \mathrm{rpm}$ Is very much alike and is a doughnut configuration with a near circular kidneyshape cross-section based on the computation of the numerical algorithm developed in our earlier studies ${ }^{3}$. Figure 4 shows the initial shape of the interface in the r-z plane at $\theta=0^{\circ}$ and $180^{\circ}$; Figure 4 shows the initial profile of the liquid-vapor interface in the $r-z$ plane at $\theta=90^{\circ}$ and $270^{\circ}$; Figure 4 shows the initial profile of the liquid-vapor interface in the $r-\theta$ plane at height $z-108$ $\mathrm{cm}$; and Figure 4 shows the initial profile of the three-dimensional liquid-vapor interface. Because of page limitation, the time evolution of the threedimensional liquid-vapor interface profiles only will be illustrated in this paper.

In this study, the combined gravity gradient and three ranges of $j$ itter $\left(10^{-6}, 10^{-7}\right.$, and $\left.10^{-8} \mathrm{go}_{0}\right)$ accelerations acting on the liquid-vapor interface oscillations have been investigated.

(III-A) Combined Gravity Gradient and $10^{-8}$ go Background Jitter Accelerations Acting on Liquid-Vapor Interface Oscillations

In this case, the combined effects of gravity gradient and jitter accelerations are completely dominated by the gravity gradient acceleration when the $10^{-8}$ go and less background gravity jitter accelerations are applied simultaneously with gravity gradient acceleration (which is on the order of $10^{-7}$ go as shown in Figure 2) for the excitation of slosh waves along liquid-vapor 
interface oscillations.

Figure 5 shows the time sequence evolution of the liquid-vapor interface profiles driven by these combined accelerations. For the convenience of comparison, figures of liquid-vapor interface profiles with the same values of the time sequences chosen throughout this paper are at time $t-191,354,380$, $431,503,603,825,980,995,1050,1080$ and $1200 \mathrm{~s}$. It clearly shows that there is a series of asymmetric oscillations excited along the surface of 1iquid-vapor interface driven by asymetric gravity gradient-dominated acceleration.

Careful examination of the case of gravity gradient-dominated accelerationeffected liquid-vapor interface oscillations, gravity gradient acceleration shown in Equation (3), indicate that there are greater negative components of acceleration longitudinal to the direction aligned with spacecraft mass center to the Earth center, and smaller positive components of acceleration transverse to this direction. As we indicated in Equation (4), angle $\psi_{\mathrm{E}}$ varies with time. This phenomena shows that the gravity gradient acceleration exerted on the spacecraft is equivalent to the combination of time dependent force with turnaround direction and torsional moment acting on the spacecraft when it is orbiting around the Earth. It shows in Figures 4 and 5 that the deformation of the bubble is created by asymmetric torsional moment with a twisting force.

Figure 5 shows the time sequence evolution of the three-dimensional dynamical behavior of the interface oscillations driven by gravity gradientdominated acceleration. It indicates that the bubble (helium vapor) configurations change from axial symmetric to asymetric profiles at a plane aligned with the vector of gravity gradient acceleration. The gravity gradientdominated acceleration produces a combination of time dependent torsional movement with tidal motion of bubble oscillations in the rotating dewar when it 
is orbiting around the Earth.

(III-B) Combined Gravity Gradient and $10^{-7}$ go Background Jitter Accelerations Acting on Liquid-Vapor Interface Oscillations

In this section, the combined effects of accelerations are equally weighted by both the gravity gradient and jitter accelerations when $10^{-7}$ go background gravity jitter acceleration is jointly applied simultaneously with gravity gradient acceleration (which is on the order of $10^{-7} \mathrm{go}$ as shown in Figure 2) for the excitation of slosh waves along the liquid-vapor interface oscillations. Figure 6 shows the time sequence evolution of the dynamical behavior of the liquid-vapor interface oscillations driven by these combined accelerations. Similar to Figure 5, the same values of the time sequences are chosen. It also shows that there are a series of asymmetric oscillations excited along the surface of liquid-vapor interface driven by asymmetric equally weighted gravity gradient and jitter accelerations.

As we recorded in Figure 5, the effect of gravity gradient-dominated acceleration exerted on the spacecraft is equivalent to the combination of timedependent force with turn-around direction of torsional moment acting on the spacecraft when it is orbiting around the Earth. On the other hand, it will be show later in Section (III-C) for the case of gravity jitter-dominated acceleration exerted on the spacecraft which is equivalent to time-dependent oscillatory forces that push the bubble in the combined directions of down-and-up and leftward-and-rightward as the bubble is rotating with respect to the spacecraft rotation axis. Figure 6 exactly illustrates these combined effects of equally weighted gravity gradient and jitter accelerations which drive the bubble in a manner of combined oscillations of the twisting deformation of the bubble associated with down-and-up motions. 
A combined oscillation of twisting bubble oscillations with down-and-up bubble oscillations modified by leftward-and-rightward oscillations indicates very important characteristics of combined gravity gradient and jitter accelerations of equal weight acting on the spacecraft when it is orbiting around the Earth.

(III-C) Combined Gravity Gradient and $10^{-6}$ go Background Jitter Accelerations Acting on Liquid-Vapor Interface Oscillations

In this case, the combined effects of gravity gradient and jitter accelerations are completely dominated by the gravity fitter acceleration when the $10^{-6}$ go and higher background gravity jitter acceleration are applied simultaneously with gravity gradient acceleration (which is on the order of $10^{-7}$ go as shown in Figure 2) for the excitation of slosh wave along the liquid-vapor interface oscillations.

Figure 7 shows the time sequence evolution of the dynamical behavior of the liquid-vapor interface oscillations driven by gravity jitter-dominated acceleration of $0.1 \mathrm{~Hz}$ low jitter frequency. It clearly shows that there is a series of asymmetric oscillations excited along the surface of liquid-vapor interface driven by asymmetric gravity jitter-dominated acceleration.

Careful examination of the case of gravity jitter acceleration-dominate liquid-vapor interface oscillations, gravity jitter acceleration, shown in Equations (5) and (6) which is also illustrated in Figure 3, indicates that there is a sinusoidal oscillation longitudinal to the direction aligned with spacecraft mass center to Earth center (parallel to unit vector $\dot{r}_{c}$ shown in Figure 1 ). As we indicated in Equation (4), the angle $\psi_{E}$ varies with time. This phenomena shows that the gravity jitter acceleration exerted on the spacecraft is equivalent to time-dependent oscillatory forces which push the bubble in the 
combined directions of down-and-up (see z-component of gravity jitter acceleration shown in Figure 3) and leftward-and-rightward (see $x$-and-y components of gravity jitter accelerations shown in Figure 3 ) as the bubble is rotating with respect to the spacecraft rotation axis. The down-and-up motion of the bubble is due to the fact that $z$-component gravity jitter acceleration decreases from the maximum downward direction to the minimum downward direction, during the time period between $t=0$ to $t=300 \mathrm{~s}$, which pushes the liquid downward and the bubble upward; while the gravity jitter acceleration changes to its downward direction and magnitude from the minimum upward to the maximum upward, and then back to the minimum upward, during the time period between $t$ $300 \mathrm{~s}$ to $\mathrm{t}-900 \mathrm{~s}$, which pushes the liquid upward and the bubble downward; in the meanwhile, the gravity jitter acceleration changes to its downward direction and magnitude during the period from $t=900 \mathrm{~s}$ to $1200 \mathrm{~s}$ which repeats the half cycle story of the time period from $t=0$ to $t=300 \mathrm{~s}$. The leftward-andrightward oscillations of bubble are the results of positive and negative directions of gravity jitter accelerations in the $\mathrm{x}$ - and $\mathrm{y}$-components as that illustrated in Figure 3. The positive direction gravity jitter acceleration implies that the liquid is pushed rightward and the bubble is driven leftward. The time variations of gravity jitter accelerations, shown in Figure 3 , characterize the leftward and rightward oscillations of the bubbles shown in Figure 7.

In addition to the $0.1 \mathrm{~Hz}$ low frequency gravity fitter-dominated acceleration, $1.0 \mathrm{~Hz}$ medium frequency and $10 \mathrm{~Hz}$ high frequency gravity jitter acceleration driven liquid-vapor interface oscillations have also been investigated. The characteristics of these three ranges of gravity jitter frequencies have been well-documented in the earlier studies ${ }^{3-6}$. Because of page 
limitation, oscillations driven by the time-dependent variations of the direction of gravity fitter-dominated acceleration with medium and high frequencies will not be shown in this paper. It is also worthwhile to mention that the natural frequency of a cryogenic hellum container is much closer to the low frequency jitter acceleration than that of the medium and high frequencies. Results show that lower frequency gravity jitter-dominated acceleration contribute more in the driving of asymmetric profiles of the interface than the higher frequency gravity jitter-dominated acceleration. These results are agreeable with the earlier studies ${ }^{3-6}$.

IV. Characteristics of Slosh Wave Induced Fluctuations

In Fluid System Moment and Angular Momentum

Slosh wave induced fluctuations in the fluid system of the rotating dewar introduce time-dependent disturbances in moment and angular momentum of the spacecraft propulsion system. In this study, in addition to the existing angular velocity along the rolling axis, there are induced angular velocities along the yawing and pitching axes due to the sloshing dynamic modulated fluid motion inside the rotating container. These disturbances of angular velocities in the yawing and pitching axes readjust the angular velocity in the rolling axis.

In order to accommodate the spacecraft dynamics of yawing, pitching and rolling, the cylindrical coordinates (shown in Figure 1) of the rotating container are transformed into cartesian coordinates based on $(x, y, z)=(\operatorname{rcos} \theta$, $r \sin \theta, z)$ with $\operatorname{corresponding~velocity~} \operatorname{components}\left(\mathrm{v}_{\mathrm{x}}, \mathrm{v}_{\mathrm{y}}, \mathrm{v}_{\mathrm{z}}\right)=(\mathrm{ucos} \theta-v \sin \theta$, usin $\theta+v \cos \theta$, w). If the spacecraft is rotated with respect to the mass center at $\left(r_{c}, \theta_{c}, z_{c}\right)$ in cylindrical coordinates, the location of the mass center in cartesian coordinates becomes $\left(x_{c}, y_{c}, z_{c}\right)=\left(r_{c} \cos \theta_{c}, r_{c} \sin \theta_{c}, z_{c}\right)$. The velocity of the mass center in cylindrical and cartesian coordinates which are defined in 
the non-inertia frame is $\left(u_{c}, v_{c}, w_{c}\right)$ and $\left(v_{c, x}, v_{c, y}, v_{c, z}\right)$, respectively. Detailed mathematical derivations of induced fluid angular velocities, fluid angular momentum, and fluid moment modulated by sloshing dynamics driven by orbital accelerations are illustrated in our recent publications ${ }^{16,21}$ and will not be repeated in this paper.

(IV-A) Combined Gravity Gradient and $10^{-8}$ Background Jitter Accelerations Driven Angular Momentum Fluctuations

As we mentioned earlier, the combined effects of gravity gradient and jitter accelerations are completely dominated by the gravity gradient acceleration when the $10^{-8} \mathrm{go}$ and less background gravity jitter accelerations are applied simultaneously with gravity gradient acceleration which drive fluid system moment and angular momentum fluctuations.

Figure $8(A)$ shows the computed time variation of the fluctuations of angular momentum driven by gravity gradient-dominated acceleration. This figure shows the following results: (a) The values of angular momentum fluctuations are $\left(\Delta \mathrm{H}_{\mathrm{x}}, \Delta \mathrm{H}_{\mathrm{y}}, \Delta \mathrm{H}_{z}\right)=(2.765,2.269,5.094) 10^{5} \mathrm{~g} \cdot \mathrm{cm}^{2} / \mathrm{s}$, it clearly indicates $\Delta \mathrm{H}_{z}>\Delta \mathrm{H}_{\mathrm{x}}$ $>\Delta H_{y}$. The maximum absolute values of angular moment are $\operatorname{Max}\left(\left|H_{x}\right|,\left|H_{y}\right|,\left|H_{z}\right|\right)$ $=(1.57,1.30,4.166) 10^{5} \mathrm{~g} \cdot \mathrm{cm}^{2} / \mathrm{s}$. It also indicates $\left|\mathrm{H}_{z}\right|>\left|\mathrm{H}_{x}\right|>\left|\mathrm{H}_{y}\right|$.

The initial values of $H_{x}, H_{y}$ and $H_{z}$ start from zero in non-inertia frame while that of $\mathrm{H}_{2}$ starts from a non-zero value in inertia frame. This is due to the fact that $\mathrm{H}_{2}$ starts with a spinning speed of $0.1 \mathrm{rpm}$ while there is no spinning velocity along $x$ and $y$ axes at the initial point in inertia frame. Variations of oscillation frequency of $\mathrm{H}_{2}$ are a reflection of spinning speed with $0.1 \mathrm{rpm}$ in which the peak values are at 300,600, 900 and $1200 \mathrm{~s}$ and valley values are at 150,750 and $1050 \mathrm{~s}$. (d) Variation amplitudes of $\mathrm{H}_{\boldsymbol{x}}$ and $\mathrm{H}_{\mathbf{y}}$ are mainly caused by gravity gradient acceleration and are smaller than that of $\mathrm{H}_{2}$ 
because there is no spinning motion applied on the $x$ - and $y$ - axes.

Figures $9(A), 9(B)$ and $9(C)$ show variations of fluid moments due to sloshing dynamics driven by gravity gradient-dominated acceleration along the $x$, $y$ and $z$ axes, respectively. The values of fluid moment fluctuations are $\left(\Delta M_{x}\right.$, $\left.\Delta \mathrm{M}_{\mathrm{y}}, \Delta \mathrm{M}_{2}\right)=(544,526,1085)$ dyne $\mathrm{cm}$. The maximum absolute values of fluid moment are $\operatorname{Max}\left(\left|M_{x}\right|,\left|M_{y}\right|,\left|M_{z}\right|\right)=(299,285,580)$ dyne $\cdot \mathrm{cm}$. It shows $\Delta M_{z}>\Delta M_{x}$ $>\Delta M_{y}$ and $\left|M_{z}\right|>\left|M_{x}\right|>\left|M_{y}\right|$. Characteristics of the fluctuations of fluid moments driven by gravity gradient-dominated acceleration draw four point conclusions similar to those drawn for the fluctuations of angular momentum of fluid system shown earlier.

Figure $10(\mathrm{~A})$ shows time fluctuations of the locations of bubble mass centers of the rotating container due to slosh waves excited by gravity gradientdominated acceleration. The values of bubble mass center fluctuations are $\left(\Delta \mathrm{x}_{\mathrm{c}}\right.$, $\left.\Delta y_{c}, \Delta z_{c}\right)=(4.54,2.83,2.32) \mathrm{cm}$. It shows $\Delta x_{c}>\Delta y_{c}>\Delta z_{c}$ for bubble mass center fluctuations driven by gravity gradient-dominated acceleration.

(IV) - B Combined Gravity Gradient and $10^{-7}$ go Background Jitter Accelerations Driven Angular Momentum Fluctuations

As we mentioned earlier, the combined effects of accelerations are equally weighted by both the gravity gradient and jitter accelerations when $10^{-7}$ g。 background gravity jitter acceleration is jointly applied simultaneously with gravity gradient acceleration which drive fluid system moment and angular momentum fluctuations.

Fluctuations of angular momentum, fluid moment and bubble mass center driven by combined acceleration for gravity jitter acceleration with frequencies of $0.1,1.0$ and $10 \mathrm{~Hz}$ have been investigated. Because of page limitation, the results of fluctuations driven by combined acceleration for $0.1 \mathrm{~Hz}$ gravity jitter 
frequency only will be presented in this study. Figure $8(B)$ shows the computed time variation of the fluctuations of angular momentum driven by equally weighted gravity gradient and jitter accelerations with frequency of $0.1 \mathrm{~Hz}$. The values of angular momentum fluctuations are $\left(\Delta \mathrm{H}_{\mathrm{x}}, \Delta \mathrm{H}_{\mathrm{y}} \Delta \mathrm{H}_{\mathrm{z}}\right)=(3.266,2.55,10.308) 10^{5}$ $\mathrm{g} \cdot \mathrm{cm}^{2} / \mathrm{s}$ and the maximum absolute values of angular moment are $\operatorname{Max}\left(\left|\mathrm{H}_{\mathbf{x}}\right|,\left|\mathrm{H}_{\mathbf{y}}\right|\right.$. $\left.\left|H_{z}\right|\right)=(1.666,1.423,9.472) 10^{5} \mathrm{~g} \cdot \mathrm{cm}^{2} / \mathrm{s}$. It shows $\Delta H_{z}>\Delta H_{x}>\Delta H_{y}$ and $\left|H_{z}\right|>$ $\left|H_{x}\right|>\left|H_{y}\right|$. The results are very much similar to the four points conclusions driven for the angular momentum fluctuations driven by gravity gradient-dominated acceleration. It also shows that equally weighted gravity gradient and jitter accelerations have excited greater magnitudes and fluctuations of angular momentum than those driven by gravity gradient-dominated acceleration.

Figures $11(\mathrm{~A}), 11(\mathrm{~B})$ and $11(\mathrm{C})$ show variations of fluid moments due to slosh waves excited by equally weighted gravity gradient and jitter accelerations with frequency of $0.1 \mathrm{~Hz}$ along the $x, y$ and $z$ axes, respectively. The values of fluid moment fluctuations are $\left(\Delta M_{x}, \Delta M_{y}, \Delta M_{z}\right)=(838,800,1797)$ dyne $\cdot \mathrm{cm}$ and the maximum absolute values of fluid moment are $\operatorname{Max}\left(\left|M_{x}\right|,\left|M_{y}\right|,\left|M_{z}\right|\right)-(485,468$, 965) dyne $\mathrm{cm}$. It shows $\Delta M_{z}>\Delta M_{x}>\Delta M_{y}$ and $\left|M_{z}\right|>\left|M_{x}\right|>\left|M_{y}\right|$. Characteristics of the fluctuations of fluid moments driven by equally weighted gravity gradient and $j$ itter accelerations draw four point conclusions similar to those drawn for the fluctuations of angular momentum of fluid systems driven by gravity gradientdominated acceleration shown earlier. It also shows that equally weighted combined gravity gradient and jitter acceleration have excited greater magnitudes and fluctuations of fluid moment than that driven by gravity gradient-dominated acceleration.

Figure 10(B) shows time fluctuations of the locations of bubble mass centers of the rotating container due to slosh waves excited by equally weighted 
gravity gradient and jitter accelerations. The values of bubble mass center fluctuations are $\left(\Delta \mathrm{x}_{c}, \Delta \mathrm{y}_{\mathrm{c}}, \Delta \mathrm{z}_{\mathrm{c}}\right)=(7.63,6.27,11.48) \mathrm{cm}$. A comparison of Figures $10(\mathrm{~A})$ and $10(\mathrm{~B})$ for bubble mass center fluctuations driven between gravity gradient-dominated acceleration and equally weighted gravity gradient and jitter accelerations provide the following conclusions: (a) Torsional moment-and twisting-force equivalent gravity gradient-dominated acceleration exerted on the rotating dewar container produces smaller values and fluctuations of angular momentum and fluid moment than that driven by equally weighted gravity gradient and fitter accelerations. (b) Dynamics of bubble (liquid-vapor interface) driven by torsional moment-and-twisting force-equivalent gravity gradientdominated acceleration produces bubble mass center fluctuations of $\Delta x_{c}>\Delta y_{c}>$ $\Delta z_{c}$ while up and down oscillations of bubble driven by equally weighted gravity gradient and jitter accelerations induces bubble center fluctuations of $\Delta z_{c}>\Delta x_{c}$ $>\Delta y_{c}$. (c) A comparison of the values of bubble mass center fluctuations shows that the fluctuations of bubble mass center induced by equally weighted gravity gradient and jitter accelerations are greater than those induced by gravity gradient-dominated acceleration. (d) Fluctuations of both $x_{c}$ and $y_{c}$ start from zero while that of the $z_{c}$ starts from a non-zero value at the middle point of the height of partially filled liquid container.

(IV-C) Combine Gravity Gradient and $10^{-6}$ go Background Jitter Accelerations Driven Angular Momentum Fluctuations

As we mentioned earlier, the combined effects of gravity gradient and jitter accelerations are completely dominated by the gravity jitter acceleration when the $10^{-6} g_{0}$ and higher background jitter acceleration are applied simultaneously with gravity gradient acceleration which drive fluid system moment and angular momentum fluctuations. 
Figure $8(C)$ shows the computed time variation of the fluctuations of angular momentum driven by gravity jitter-dominated acceleration with frequency of $0.1 \mathrm{~Hz}$. The values of angular momentum fluctuations are $\left(\Delta \mathrm{H}_{\mathrm{x}}, \Delta \mathrm{H}_{\mathrm{y}} \Delta \mathrm{H}_{\mathrm{z}}\right)-$ $(16.013,6.753,29.164) 10^{5} \mathrm{~g} \cdot \mathrm{cm}^{2} / \mathrm{s}$ and the maximum absolute values of angular moment are $\operatorname{Max}\left(\left|\mathrm{H}_{x}\right|,\left|\mathrm{H}_{y}\right|,\left|\mathrm{H}_{z}\right|\right)=(8.667,3.986,29.165) 10^{5} \mathrm{~g} \cdot \mathrm{cm}^{2} / \mathrm{s}$. It shows $\Delta H_{z}>\Delta H_{x}>\Delta H_{y}$ and $\left|H_{z}\right|>\left|H_{x}\right|>\left|H_{y}\right|$. A comparison of Figures $8(A), 8(B)$ and 8 (C) for angular momentum fluctuations driven by gravity gradient-dominated acceleration and gravity jitter-dominated acceleration shows that gravity gradient-dominated acceleration have excited smaller magnitudes and fluctuations of angular momentum than that driven by gravity jitter-dominated acceleration.

Figures $12(\mathrm{~A}), 12(\mathrm{~B})$ and $12(\mathrm{C})$ show variations of fluid moments due to slosh waves excited by gravity jitter-dominated acceleration with frequency of $0.1 \mathrm{~Hz}$ along the $x, y$ and $z$ axes, respectively. The values of fluid moment fluctuations are $\left(\Delta M_{x}, \Delta M_{y}, \Delta M_{z}\right)=(2373,2346,5423)$ dyne $\mathrm{cm}$ and the maximum absolute values of fluid moment are $\operatorname{Max}\left(\left|M_{x}\right|,\left|M_{y}\right|,\left|M_{z}\right|\right)=(1282,1235,3043)$ dyne $\cdot \mathrm{cm}$. It shows $\Delta M_{z}>\Delta M_{x}>\Delta M_{y}$ and $\left|M_{z}\right|>\left|M_{x}\right|>\left|M_{y}\right|$. Characteristics of the fluctuations of fluid moments driven by gravity jitter-dominated acceleration draw four point conclusions similar to those drawn for the fluctuations of angular momentum of fluid systems driven by gravity gradient-dominated acceleration shown earlier. It also shows that gravity gradient-dominated acceleration have excited smaller magnitudes and fluctuations of fluid moment than that driven by gravity jitter-dominated acceleration.

Figure $10(\mathrm{C})$ shows time fluctuations of the locations of bubble mass centers of the rotating container due to slosh waves excited by asymmetric gravity jitter-dominated acceleration. The values of bubble mass center fluctuations are $\left(\Delta x_{c}, \Delta y_{c}, \Delta z_{c}\right)=(17.28,12.2,33.83) \mathrm{cm}$. A comparison of 
Figures $10(\mathrm{~A}), 10(\mathrm{~B})$ and $10(\mathrm{C})$ for bubble mass center fluctuations driven between gravity gradient-dominated and gravity jitter-dominated accelerations provides the following conclusions: (a) Torsional moment-and twisting force-equivalent gravity gradient-dominated acceleration exerted on the rotating dewar container produces smaller values of magnitudes and fluctuations of angular momentum and fluid moment than that driven by gravity jitter-dominated acceleration. (b) Dynamics of bubble (1iquid-vapor interface) driven by torsional moment-andtwisting force-equivalent gravity gradient acceleration produces bubble mass center fluctuations of $\Delta x_{c}>\Delta y_{c}>\Delta z_{c}$ while up and down oscillations of bubble driven by gravity jitter-dominated acceleration induces bubble center fluctuations of $\Delta z_{c}>\Delta x_{c}>\Delta y_{c}$. (c) A comparison of the values of bubble mass center fluctuations show that fluctuations of bubble mass center induced by gravity jitter-dominated acceleration is greater than that induced by gravity gradient-dominated acceleration. (d) Fluctuations of both $x_{c}$ and $y_{c}$ start from zero while that of the $z_{c}$ starts from a non-zero value at the middle point of the height of partially filled liquid container. (e) Fluctuation magnitudes of $x_{c}$, $y_{c}$ and $z_{c}$ assoclated with higher frequency $j$ itter are lower than those of the frequencles associated with lower jitter frequency for bubble mass center fluctuations driven by gravity jitter-dominated acceleration.

V. Discussion and Conclusion

The dynamical behavior of cryogenic liquid systems in particular, capillary effect governed liquid-vapor interface disturbances affected by the asymmetric combined gravity gradient and jitter accelerations at three different ranges of background gravity, with an example applicable to the GP-B Spacecraft dewar have been carried out by numerically computing the three-dimensional Navier-Stokes equations subject to the initial and boundary conditions. As gravity gradient 
acceleration is pre-determined if the orbit of the spacecraft is fixed, the ranges of background gravity $j i t t e r$ acceleration will be different with various ranges of environmental drag due to the sources of residual acceleration, solar wind environment during solar minimum and maximum cycles, etc. Gravity jitter accelerations with three ranges of background gravity at $10^{-6}, 10^{-7}$ and $10^{-8}$ go are chosen in this study.

Induced angular velocities along the rolling, yawing and pitching axes caused by slosh wave excitation driven by gravity gradient-dominated and gravity jitter-dominated accelerations have been investigated. Results show that there is a series of large amplitude fluctuation of angular momentums and fluid moments along the rolling, yawing and pitching axes. In particular, slosh waves excited by the asymmetry gravity gradient-dominated and gravity jitter-dominated accelerations contribute greatly to the large amplitude fluctuations of angular momentum and fluid moment along the rolling axis of the spacecraft. The study of bubble mass center fluctuations driven by gravity gradient-dominated and gravity jitter-dominated accelerations show that gravity gradient-dominated acceleration drives greater bubble mass center fluctuations in the pitching axis while gravity jitter-dominated acceleration induces greater bubble mass fluctuations in the rolling axis.

The time-dependent variations in the direction of gravity gradient and gravity jitter-dominated accelerations imposed on the spacecraft will change and modify the asymmetry distribution of liquid-vapor in the rotating dewar container. This means that fluid moment, angular momenturn and bubble mass center will be deeply disturbed by the presence of asymmetric fluctuations in liquidvapor interface. In this study, the differences in gravity gradient-dominated acceleration and gravity jitter-dominated acceleration induced slosh waves and 
how these slosh wave excitation affected the fluctuations in angular momentum, fluid moment and bubble mass center disturbances have been studied. A better understanding of sloshing dynamics modulated fluctuations in angular momentum, fluid moment and bubble mass center distributions affected by different levels of orbital accelerations are extremely important for the development of sophisticated attitude and guidance control techniques to assure the proper operation of very complicated scientific spacecraft such as GP-B

\section{Acknowledgement}

The authors appreciate the support received from the National Aeronautics and Space Administration through the NASA Grant NAG8-938 and NASA Contract NAS838609/Delivery Order No. 103. They would like to express their gratitude to Richard A. Potter of NASA/Marshall Space Flight Center for the stimulating discussions during the course for the present study. 
1. Wilkinson, D. T., Bender, P. L., Eardley, D. M., Gaisser, T. K., Hartle, J. B., Israel, M. H., Jones, L. W., Partridge, R. B., Schramm, D. N., Shapiro, I. I., Vessort, R. F. C., and Wagoner, R. V., Gravitation, Cosmology and Cosmic-Ray Physics, Physics Today, 39, 43-46, 1986.

2. Kamotani, Y., Prasad, A., and Ostrach, S., Thermal Convection in an Enclosure Due to Vibrations Aboard a Spacecraft, AIAA Journal, 19, 511-516, 1981.

3. Hung, R. J., Lee, C. C., and Leslie, F. W., Response of Gravity Level Fluctuations on the Gravity Probe-B Spacecraft Propellant System, Journal of Propulsion and Power, $7,556-564,1991$.

4. Hung, R. J., Lee, C. C., and Leslie, F. W., Effect of the Baffle on the Spacecraft Fluid Propellant Viscous Stress and Moment Fluctuations, Transaction of the Japan Society for Aeronautical and Space Sciences, 35 , $187-207,1993$.

5. Hung, R. J., and Shyu, K. L., Space-Based Cryogenic Liquid Hydrogen Reorientation Activated by Low Frequency Impulsive Reverse Thruster of Geyser Initiation, Acta Astronautica, 25, 709-719, 1991.

6. Hung, R. J., and Shyu, K. I., Constant Reverse Thrust Activated Reorientation of Liquid Hydrogen with Geyser Initiation, Journal of Spacecraft and Rockets, $\underline{29}, 279-285,1992$.

7. Leslie, F. W., Measurements of Rotating Bubble Shapes in a Low Gravity Environment, Journal of Fluid Mechanics, 161, 269-279, 1985.

8. Mason, P., Collins, D., Petrac, D., Yang, L., Edeskuty, F., Schuch, A., and Williamson, K., The Behavior of Superfluid Helium in Zero Gravity, Proc. 7th International Cryogenic Engineering Conferences, Surrey, England, 
Science and Technology Press, 1978.

9. Avduyevsky, V. S. (editor), Scientific Foundations of Space Manufacturing, MIR, Moscow, USSR, 1984.

10. Forward, R. L., Flattening Space-Time Near the Earth, Physical Review, Series (A), 26, $735-744,1982$.

11. Misner, C. W., Thorne, K. S., and Wheeler, J. A., Gravitation, W. H. Freeman and Co., San Francisco, CA, pp. 1-1279, 1973.

12. Hung, R. J., and Pan, H. L., Differences in Gravity Gradient and Gravity Jitter-Excited Slosh Waves in Microgravity, Transactions of the Japan Society for Aeronautical and Space Sciences, 36, 153-169, 1993.

13. Hung, R. J., Pan, H. L., and Leslie, F. W., Gravity Gradient or Gravity Jitter Induced Viscous Stress and Moment Fluctuations in Microgravity, Fluid Dynamics Research, in press, 1994.

14. Hung, R. J., and Pan, H. L., Asymmetric Slosh Wave Excitation in LiquidVapor Interface Under Microgravity, Acta Mechanica Sinica, 9(4), 298-311, 1993.

15. Hung, R. J., and Leslie, F. W., Bubble Shapes in Liquid-Filled Rotating Container Under Microgravity, Journal of Spacecraft and Rockets, 25, 70-74, 1988.

16. Hung, R. J., Pan, H. L., and Leslie, F. W., Fluid System Angular Momentum and Moment Fluctuations Driven by Gravity Gradient or Gravity Jitter in Microgravity, Journal of Flight Sciences and Space Research (ZFW), in press, 1994.

17. Harlow, F. H., and Welch, F. E., Numerical Calculation of Time-Dependent Viscous Incompressible Flow of Fluid with Free Surface, Physics of Fluids, $\underline{8}, 2182-2189,1965$. 
18. Patanker, S. V., Numerical Heat Transfer and Fluid Flow, Hemisphere-McGrawHill, New York, NY, pp. 197, 1980.

19. Patanker, S. V., and Spalding, S. D., A Calculation Procedure for Heat, Mass and Momentum Transfer in Three Dimensional Parabolic Flows, International Journal of Heat Mass Transfer, 15, 1787-1805, 1972.

20. Spalding, D. B. A Novel Finite-Difference Formulation for Differential Expressions Involving Both First and Second Derivatives, International Journal of Numerical Methods in Engineering, 4, 551-559, 1972.

21. Hung, R. J., Lee, C. C., and Leslie, F. W., Effect of the Baffle on the Asymetric Gravity Jitter Excited Slosh Waves and Spacecraft Moment and Angular Momentum Fluctuations, Journal of Aerospace Engineering, 207, 105 $120,1993$.

22. Hung, R. J., Lee, C. C., and Leslie, F. W., Spacecraft Dynamical Distribution of Fluid Stresses Activated by Gravity Jitter Induced Slosh Waves, Journal of Guidance, Control and Dynamics, 15, 817-824, 1992. 
Figure Captions

Figure 1 Coordinate system for the computation of gravity gradient acceleration, $\mathrm{O}_{\mathrm{s}}$ is the location of geometric center of cylinder. Direction of $\hat{r}_{c}$ points toward Earth center. $\left(x^{\prime}, y^{\prime}, z^{\prime}\right)$ denotes absolute coordinate system, while $(x, y, z)$ illustrates non-inertia frame spacecraft bound coordinate system.

Figure 2 Time variation of gravity gradient acceleration acting on fluid mass located at $(r, \theta, z)-(40 \mathrm{~cm}, \pi / 4,10 \mathrm{~cm})$ for turn-around period of $1200 \mathrm{~s}$ with rotating speed of $0.1 \mathrm{rpm}$. (A) Along $x$-direction; (B) Along y-direction; (C) Along z-direction.

Figure 3 Time variation of gravity jitter acceleration acting on the fluid system under background gravity of $10^{-8}, 10^{-7}$ and $10^{-6} \mathrm{go}$, rotating speed of $0.1 \mathrm{rpm}$, turn-around period of $1200 \mathrm{~s}$ and gravity jitter frequency of $0.1 \mathrm{~Hz}$. (A) Along $x$-direction; (B) Along $y$-direction; (C) Along $z$-direction.

Figure 4 Initial profiles of liquid-vapor interface for Gravity Probe-B module of rotating dewar under background gravity of $10^{-7} \mathrm{go}$, rotating speed of $0.1 \mathrm{rpm}$ and direction of background gravity at $\psi_{E}=0^{\circ}$. (A) In $r-z$ plane at $\theta-0^{\circ}$ and $180^{\circ}$, (B) In $r-\theta$ plane at $\theta=90^{\circ}$ and $270^{\circ}$, (C) In $\mathrm{r}-\theta$ plane at $z=108 \mathrm{~cm}$, and (D) Three-dimensional liquidvapor interface profile.

Figure 5 Time sequence evolution of three-dimensional liquid-vapor interface oscillations for rotating dewar driven by combined gravity gradient and jitter accelerations with background gravity of $10^{-8} \mathrm{go}$. Rotating speed of dewar is $0.1 \mathrm{rpm}$, and the period of gravity direction turn-around time is $1200 \mathrm{~s}$. 
Figure 6 Time sequence evolution of three-dimensional liquid-vapor interface oscillations for rotating dewar driven by combined gravity gradient and $\mathrm{jitter}$ accelerations of $0.1 \mathrm{~Hz}$ frequency, background gravity of $10^{-7}$ go with the period of gravity direction turn-around time $1200 \mathrm{~s}$, and rotating speed of $0.1 \mathrm{rpm}$.

Figure 7 Time sequence evolution of three-dimensional liquid-vapor interface oscillations for rotating dewar driven by combined gravity gradient and $\mathrm{jitter}$ accelerations of $0.1 \mathrm{~Hz}$ frequency, background gravity of $10^{-6}$ go with the period of gravity direction turn-around time $1200 \mathrm{~s}$, and rotating speed of $0.1 \mathrm{rpm}$.

Figure 8 Time sequence of angular momentum fluctuations $\left(H_{x}, H_{y}, H_{z}\right)$ caused by the slosh wave excitation in rotating dewar with speed of $0.1 \mathrm{rpm}$ and gravity turn-around time of $1200 \mathrm{~s}$. (A) For angular momentum fluctuations driven by combined gravity gradient and fitter accelerations with background gravity of $10^{-8} \mathrm{go}$. (B) For angular momentum fluctuations driven by combined gravity gradient and $j i t t e r$ accelerations of $0.1 \mathrm{~Hz}$ gravity jitter frequency and background gravity of $10^{-7} \mathrm{go}$. (C) For angular momentum fluctuations driven by combined gravity gradient and jitter accelerations of $0.1 \mathrm{~Hz}$ frequency and background gravity of $10^{-6} \mathrm{go}$.

Figure 9 Time sequences of fluid moment fluctuations caused by slosh wave excitation driven by combined gravity gradient and jitter accelerations of $0.1 \mathrm{~Hz}$ frequency and background gravity of $10^{-8} \mathrm{~g}$ 。 in rotation speed of $0.1 \mathrm{rpm}$ and gravity turn-around time of $1200 \mathrm{~s}$. (A) For moment along $\mathrm{x}$-direction; (B) For moment along $\mathrm{y}$-direction; and (C) For moment along $z$-direction. 
Figure 10 Time sequences of bubble mass center fluctuations caused by the slosh wave excitation under gravity turn-around time of $1200 \mathrm{~s}$ and rotation speed of $0.1 \mathrm{rpm}$. (A) For bubble mass center fluctuations driven by combined gravity gradient and jitter accelerations with background gravity of $10^{-8} \mathrm{go}$. (B) For bubble mass center fluctuations driven by combined gravity gradient and jitter accelerations of $0.1 \mathrm{~Hz}$ frequency and background gravity of $10^{-7} \mathrm{~g}_{0}$; and (C) For bubble mass center combined gravity gradient and jitter accelerations of $0.1 \mathrm{~Hz}$ gravity jitter frequency and background gravity of $10^{-6} \mathrm{~g}_{0}$.

Figure 11 Time sequences of fluid moment fluctuations caused by slosh wave excitation driven by combined gravity gradient and fitter accelerations of $0.1 \mathrm{~Hz}$ frequency with background gravity of $10^{-7} \mathrm{go}$, and rotation speed of $0.1 \mathrm{rpm}$ under gravity turn-around time of 1200 s. (A) For moment along $x$-direction; (B) For moment along $y$ direction; and (C) For moment along z-direction.

Figure 12 Time sequence of fluid moment fluctuations caused by slosh wave excitation driven by combined gravity gradient and jitter accelerations of $0.1 \mathrm{~Hz}$ with background gravity of $10^{-6} \mathrm{~g}$, and rotation speed of $0.1 \mathrm{rpm}$ under gravity turn-around time of $1200 \mathrm{~s}$. (A) For moment along $\mathrm{x}$-direction; (B) For moment along $\mathrm{y}$-direction; and (C) For moment along $z$-direction. 


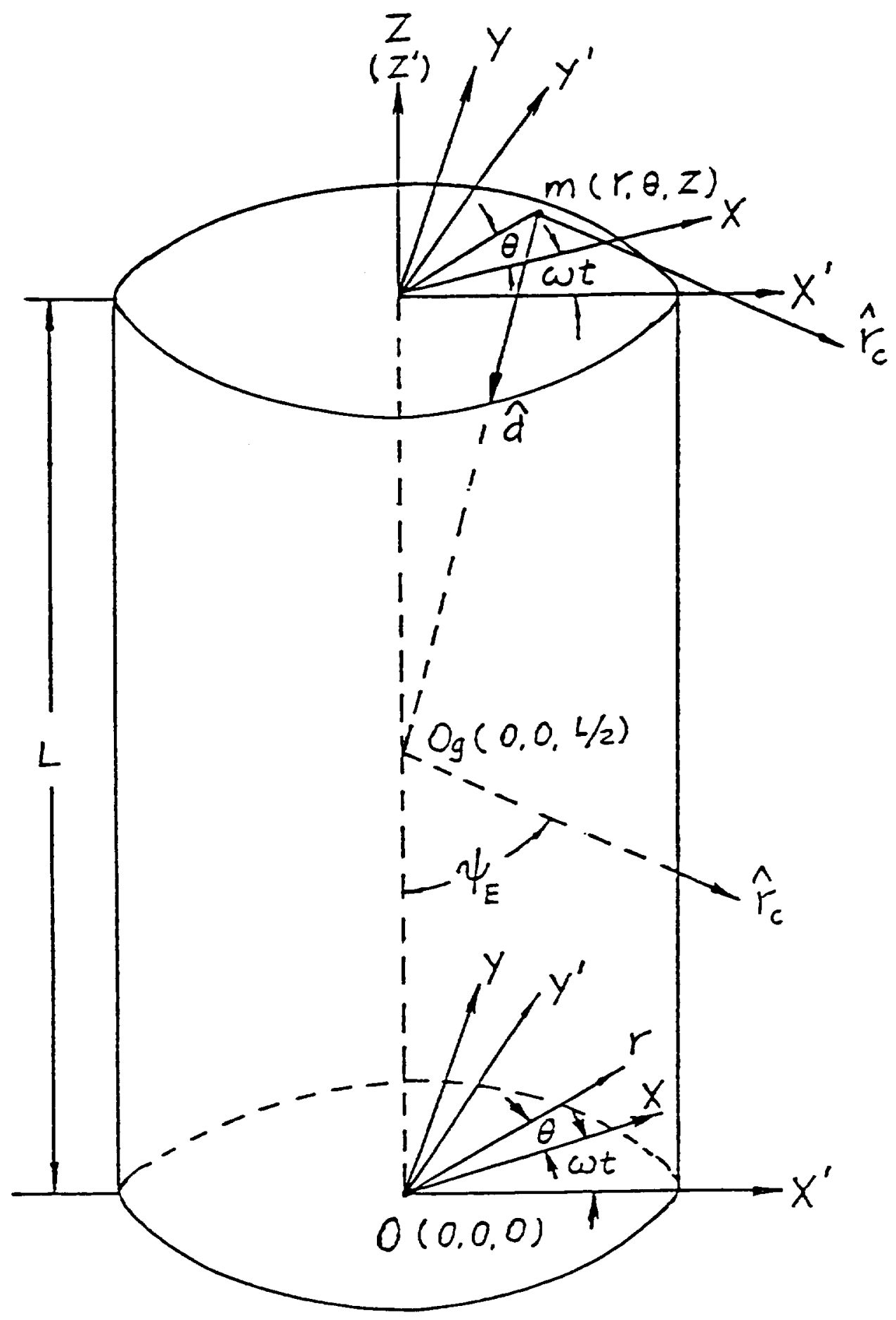

Fig. 1 
Time Variation of Gravity Gradient Acceleration Acting on the $G P-B$ Spacecraft Dewar Fluid Mass Located

$$
\begin{aligned}
& \text { at } \\
& (r, \theta, z) \\
& =(40 \mathrm{~cm}, \pi / 4, \\
& \omega=0.1 \mathrm{rpm} \\
& \tau=1200 \mathrm{~s} \\
& g_{0}=9.81 \mathrm{~m} / \mathrm{s}^{2}
\end{aligned}
$$$$
=(40 \mathrm{~cm}, \pi / 4,10 \mathrm{~cm})
$$

Fig. 2
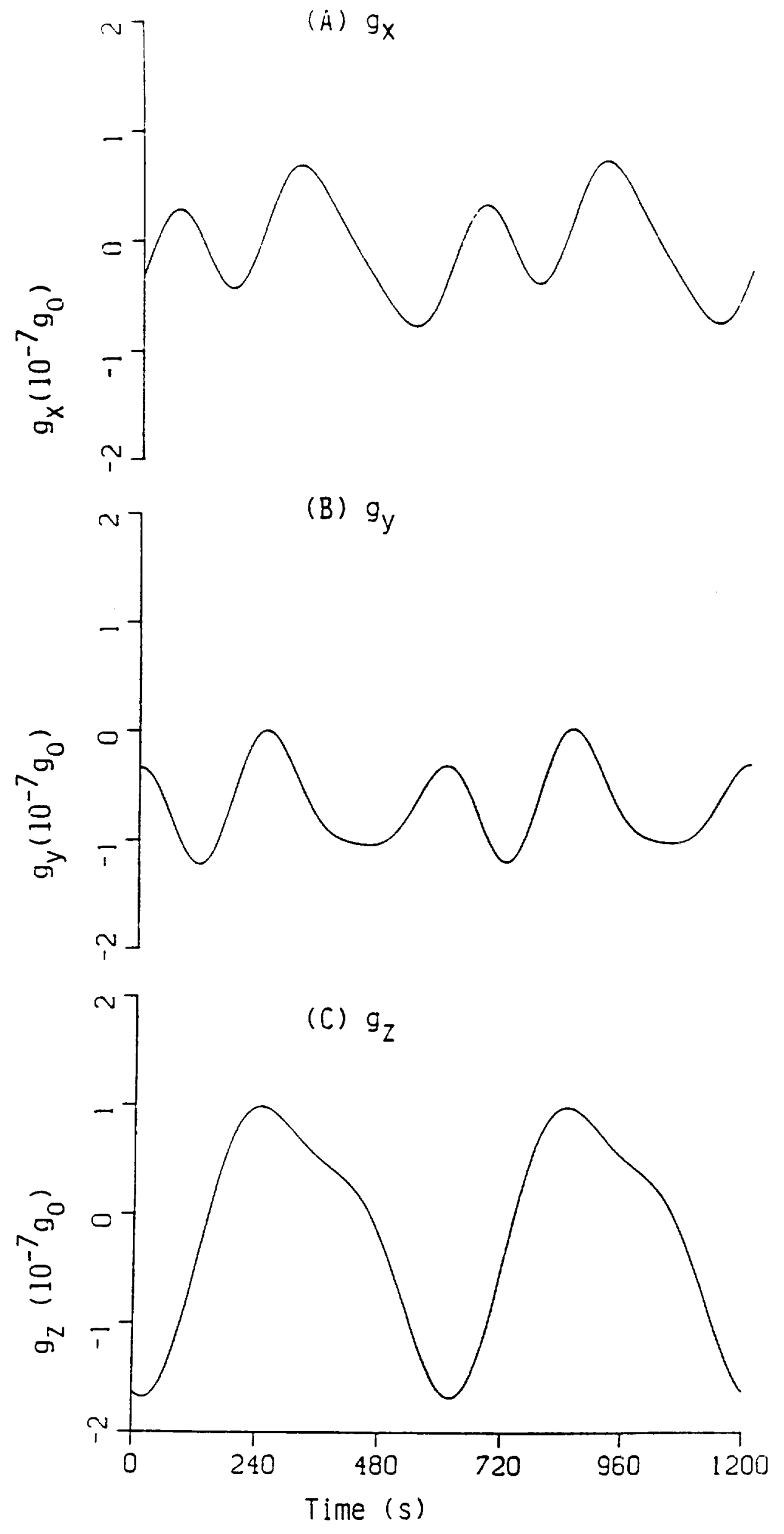
Time Variation of Gravity Jitter Acceleration Acting on Spacecraft

Fluid System

$g_{B}=\left[\begin{array}{l}10^{-6} \\ 10^{-7} \\ 10^{-8}\end{array}\right] g_{0}$

$$
\omega=0.1 \mathrm{rpm}
$$$$
f=0.1 \mathrm{~Hz}
$$$$
\tau=1200 \mathrm{~s}
$$$$
g_{0}=9.81 \mathrm{~m} / \mathrm{s}^{2}
$$
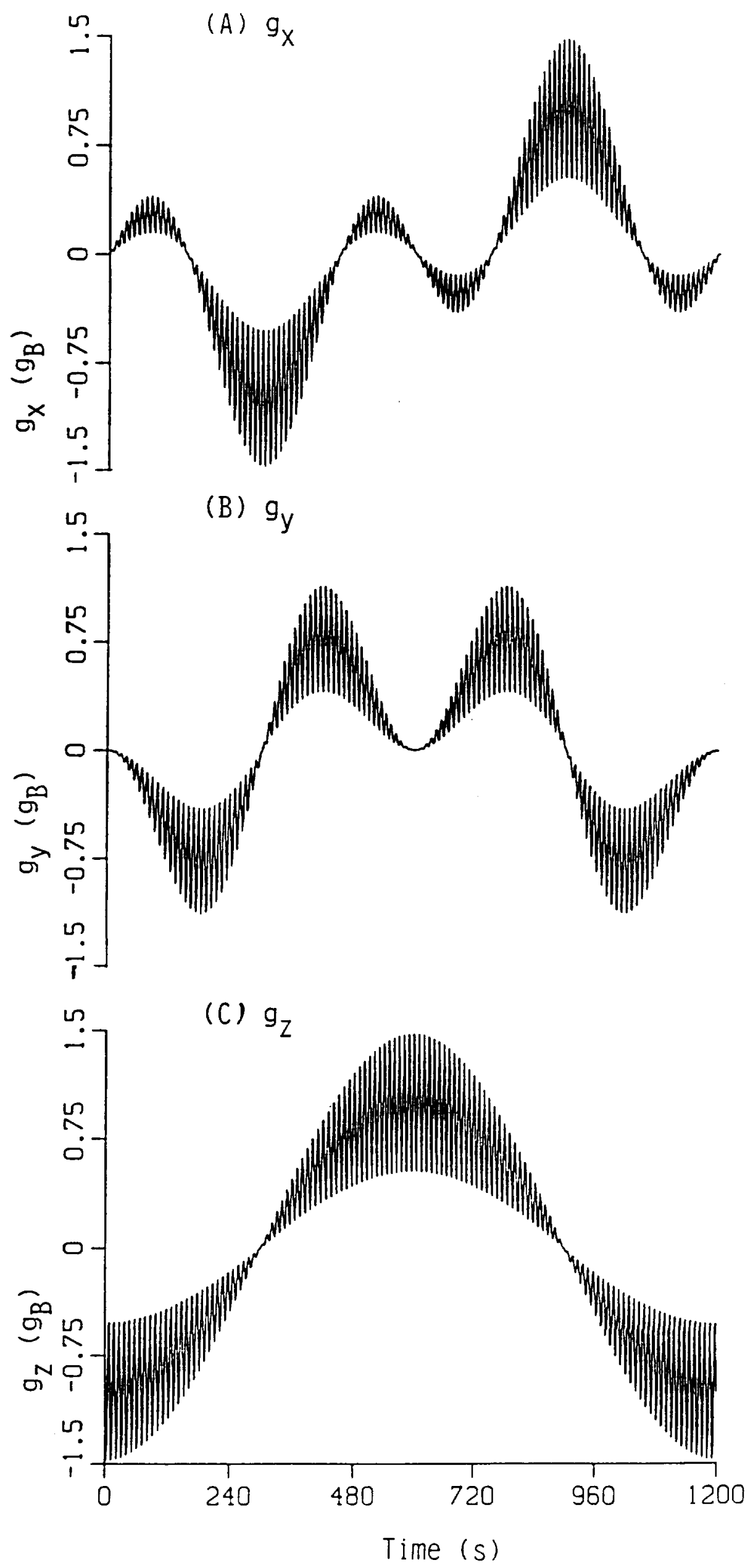
INITIAL PROFILES OF LIQUID HELIUM AND VAPOR INTERFACE

Rotating Dewar Under the Effect of Gravity Jitter

$$
g=10^{-7} g_{0}, \omega=0.1 \mathrm{rdm}, \psi_{E}=0^{\circ}, f=0,1 \mathrm{~Hz}
$$

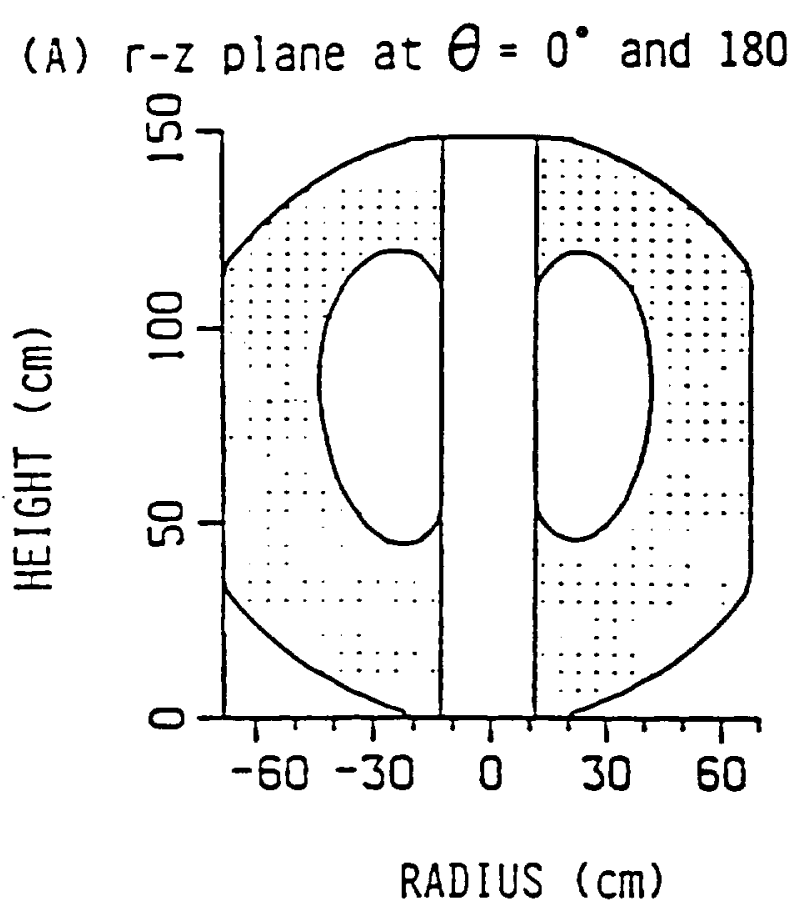

(c) $r-\theta$ plane at $z=108 \mathrm{~cm}$

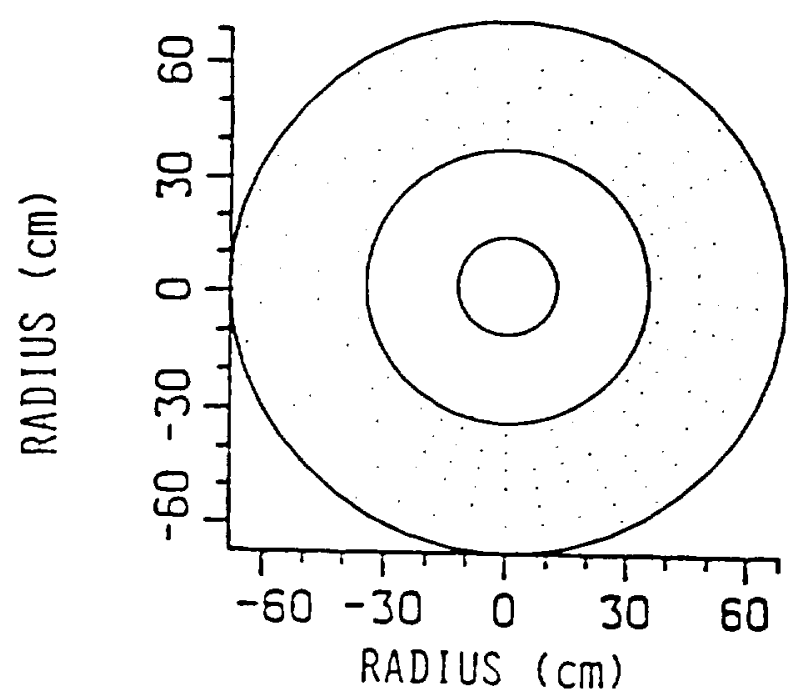

Fig. 4
(B) $r-z$ plane at $\theta=90^{\circ}$ and $270^{\circ}$

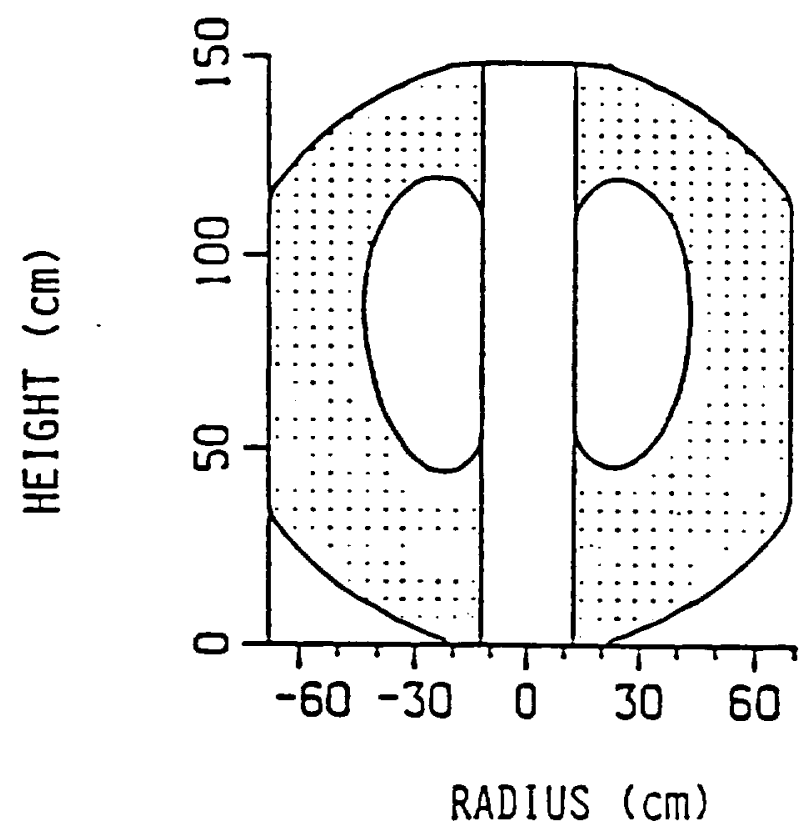

(D) Three-Dimensional Profiles

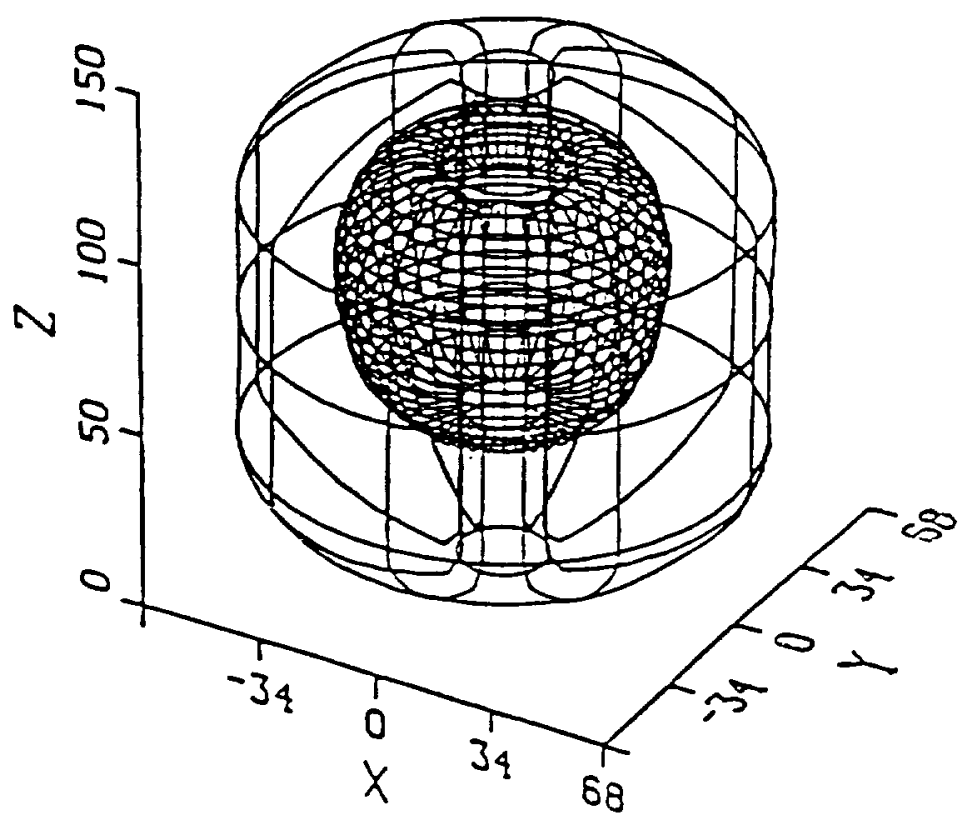


THREE DIMENSIONAL LIQUID AND VAPOR INTERFACE The Effect of Gravity Jitter and Gravity Gradient: $g=10^{-8} \mathrm{~g}$

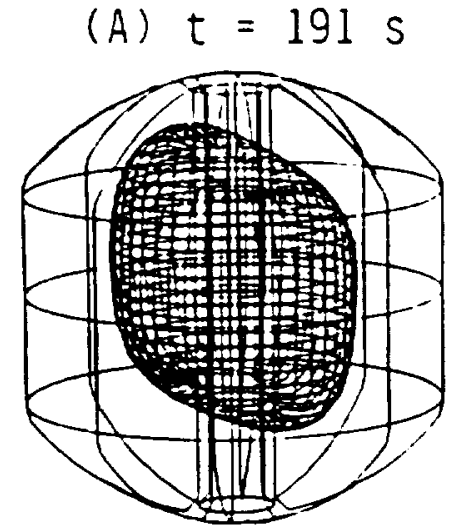

(D) $t=431 \mathrm{~s}$

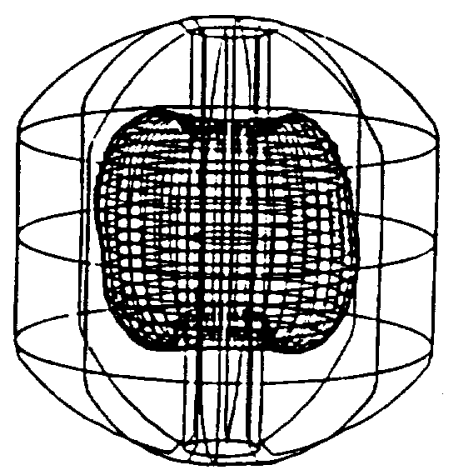

(G) $t=825 \mathrm{~s}$

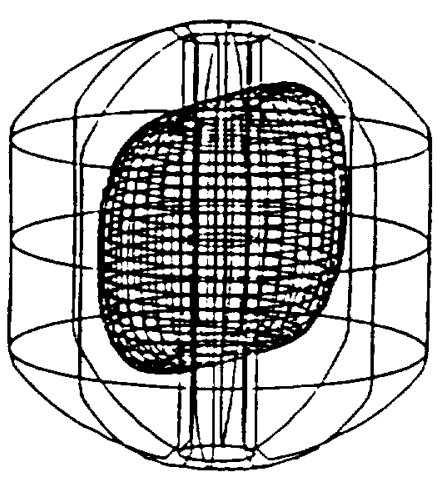

(J) $t=1050 \mathrm{~s}$

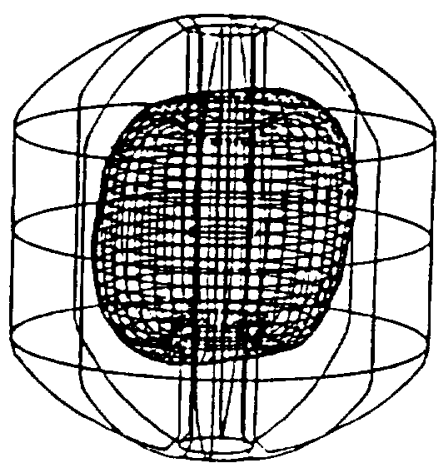

(B) $t=354 \mathrm{~s}$

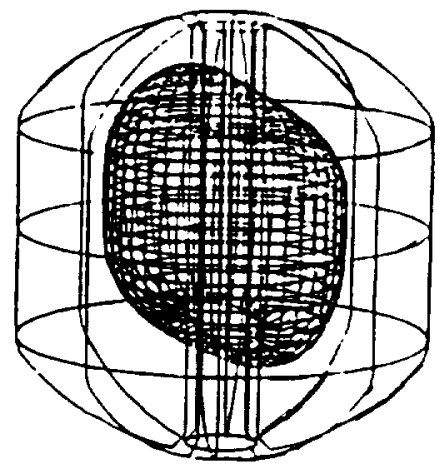

(E) $\mathrm{t}=503 \mathrm{~s}$

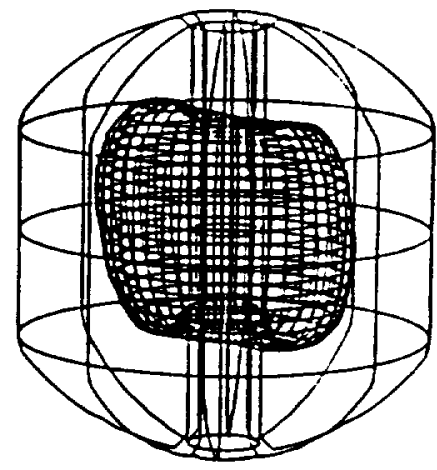

(H) $t=980 \mathrm{~s}$

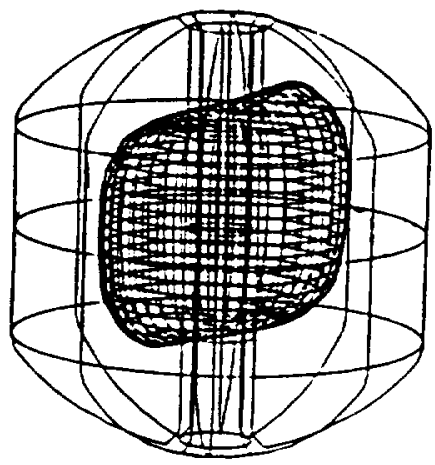

(K) $t=1080 \mathrm{~s}$

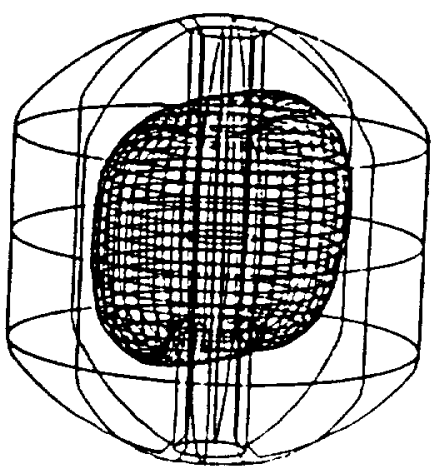

(c) $\mathrm{t}=380 \mathrm{~s}$

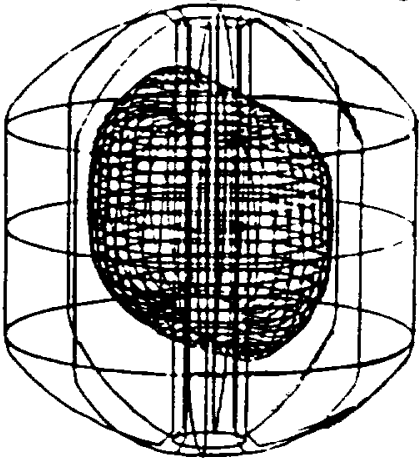

(F) $t=603 \mathrm{~s}$

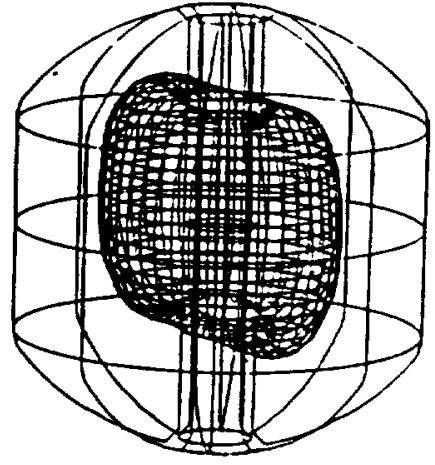

(I) $t=995 \mathrm{~s}$

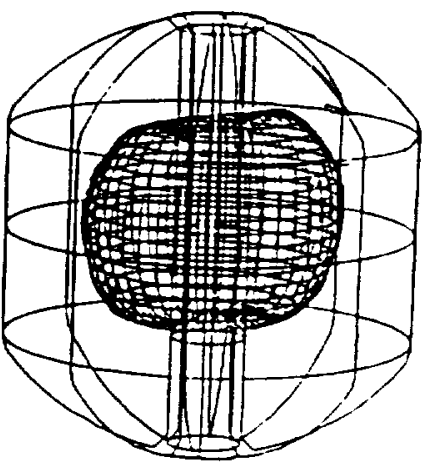

(L) $t=1200 \mathrm{~s}$

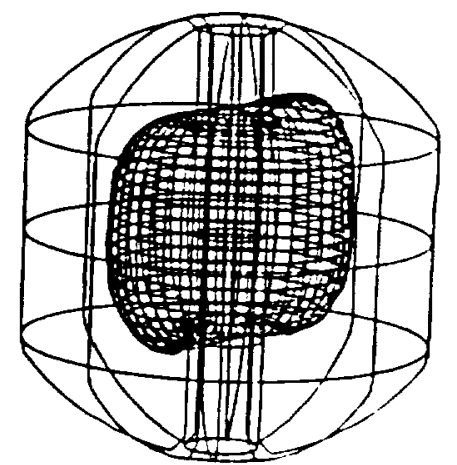

Fig. 5 
THREE DIMENSIONAL LIQUID AND VAPOR INTERFACE

The Effect of Gravity Jitter and Gravity Gradient: $g=10^{-7} \mathrm{~g}_{0}$
(A) $t=191 \mathrm{~s}$
(B) $t=354 \mathrm{~s}$
(c) $t=380 \mathrm{~s}$
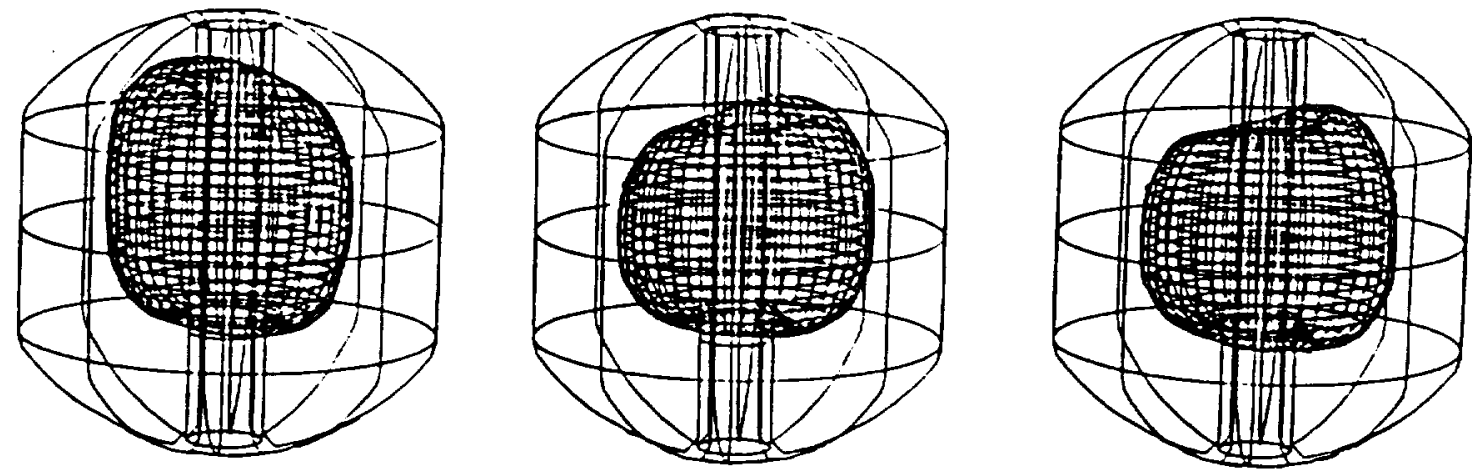

(D) $\mathrm{t}=431 \mathrm{~s}$

(E) $t=503 \mathrm{~s}$

(F) $t=603 \mathrm{~s}$
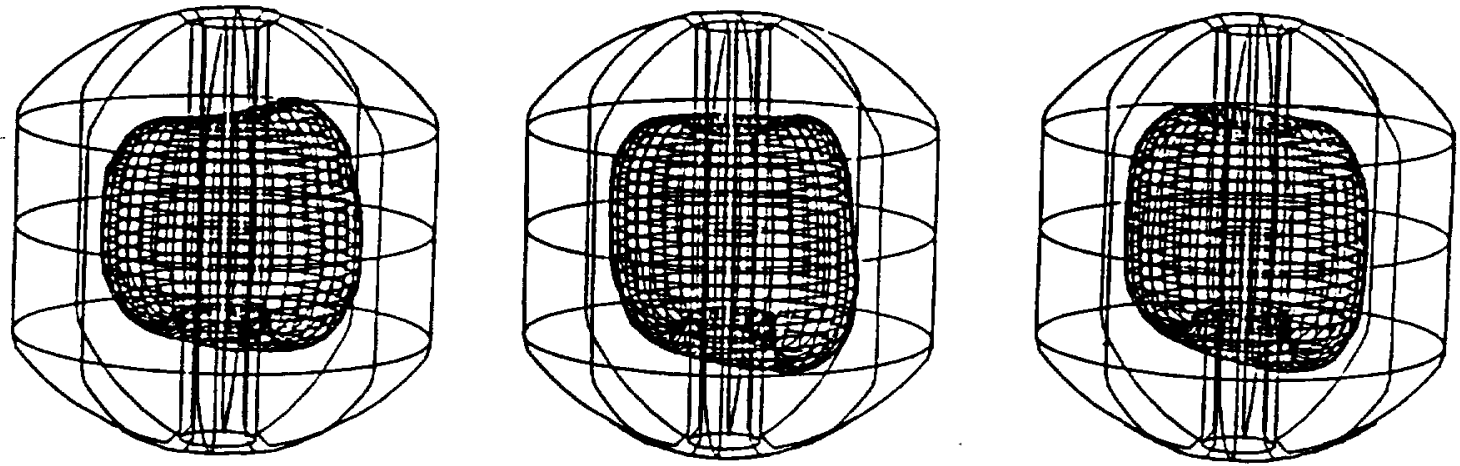

(G) $\mathrm{t}=825 \mathrm{~s}$

(H) $\mathrm{t}=980 \mathrm{~s}$

(I) $t=995 \mathrm{~s}$
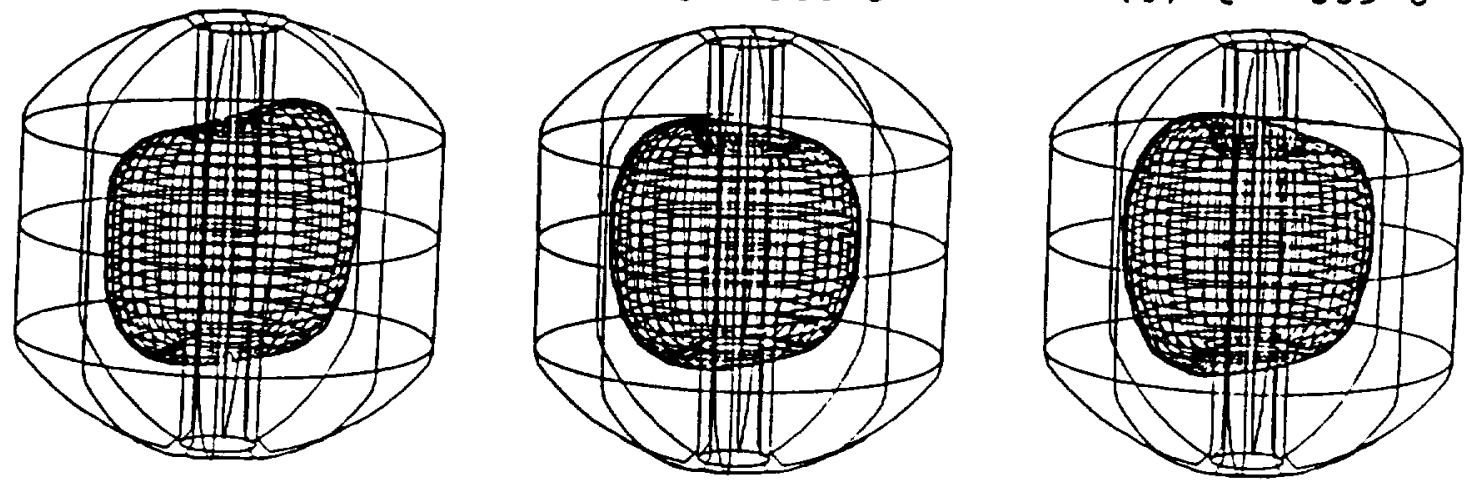

(J) $t=1050 \mathrm{~s}$

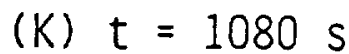

(L) $t=1200 \mathrm{~s}$

Fig. 6
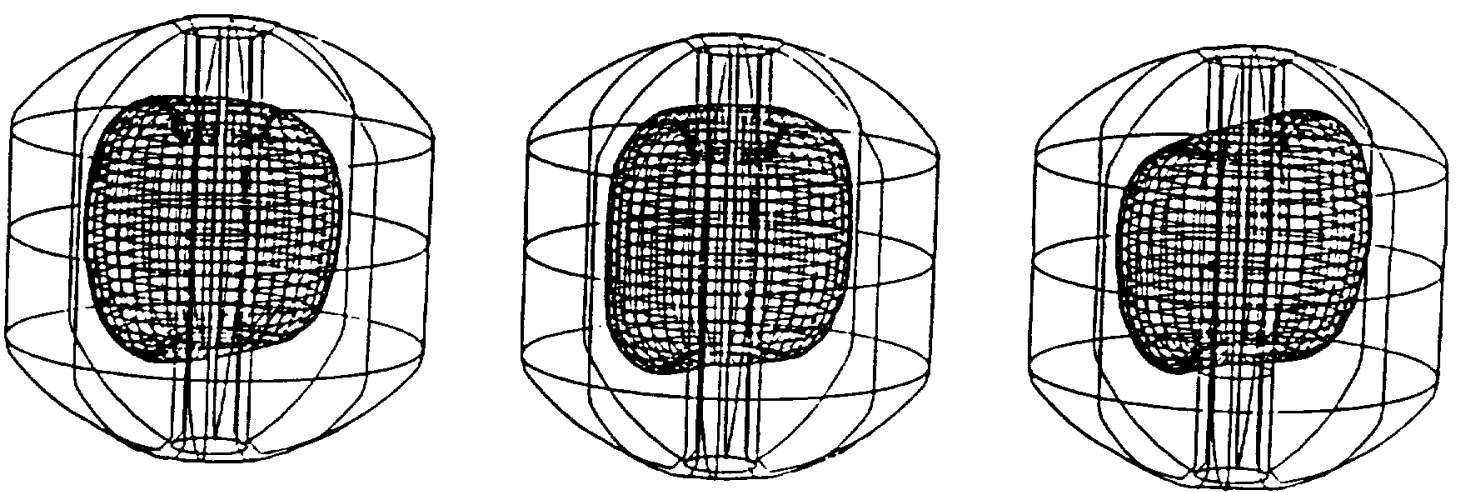
THREE DIMENSIONAL LIQUID AND VAFOR INTERFACE The Effect of Gravity Jitter and Gravity Gradient: $g=10^{-6} g_{0}$

(A) $\mathrm{t}=191 \mathrm{~s}$

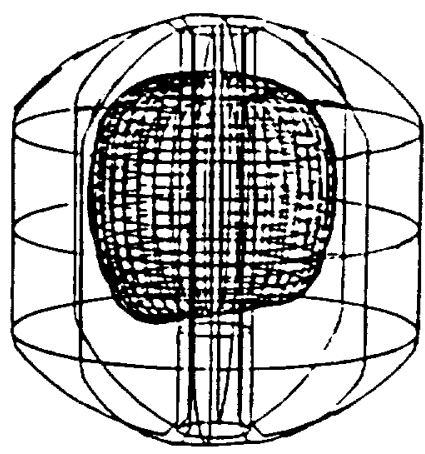

(D) $t=431 \mathrm{~s}$

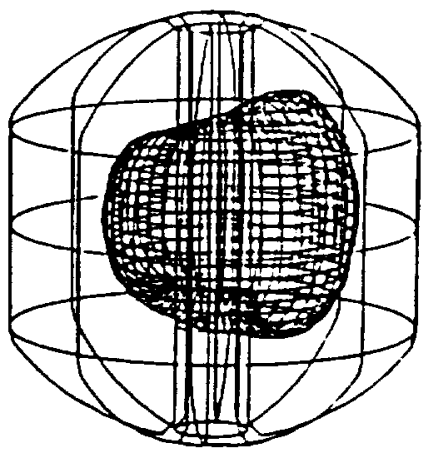

(G) $t=825 \mathrm{~s}$

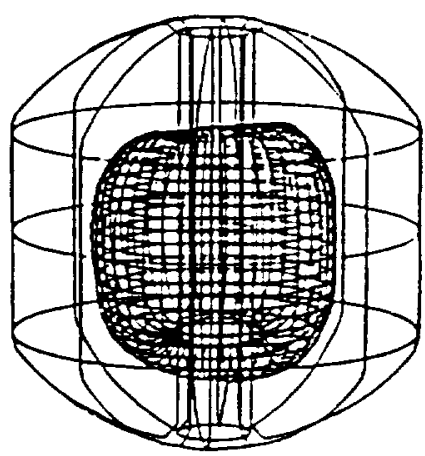

(J) $t=1050 \mathrm{~s}$

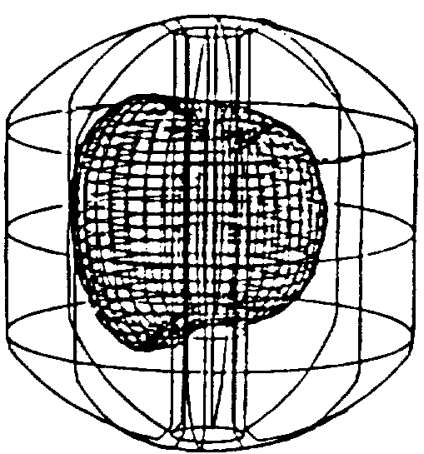

(B) $t=354 \mathrm{~s}$

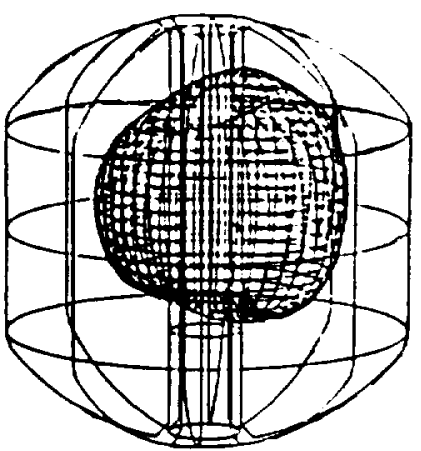

(E) $\mathrm{t}=503 \mathrm{~s}$

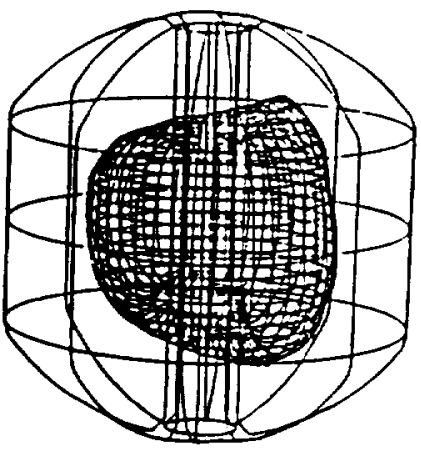

(H) $t=980 \mathrm{~s}$

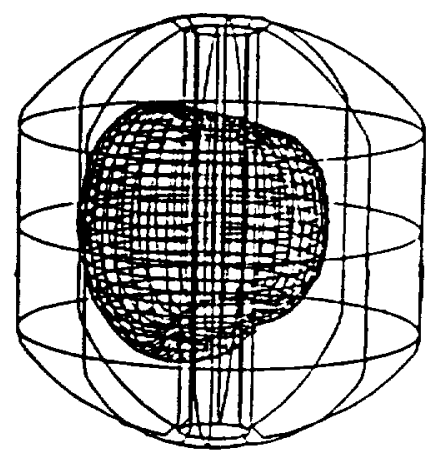

(K) $t=1080 \mathrm{~s}$

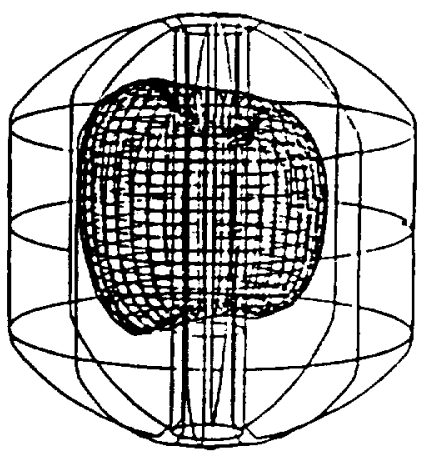

(C) $t=380 \mathrm{~s}$

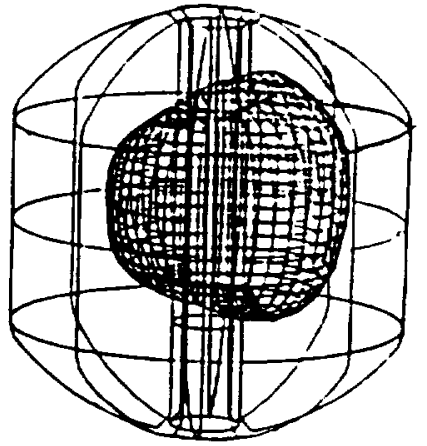

(F) $t=603 \mathrm{~s}$

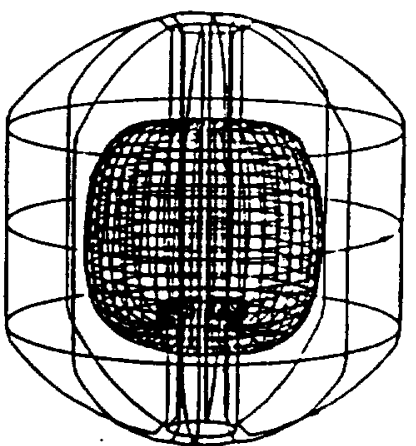

(I) $\mathrm{t}=995 \mathrm{~s}$

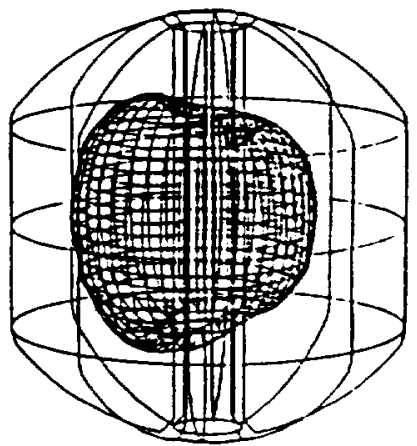

(L) $t=1200 \mathrm{~s}$

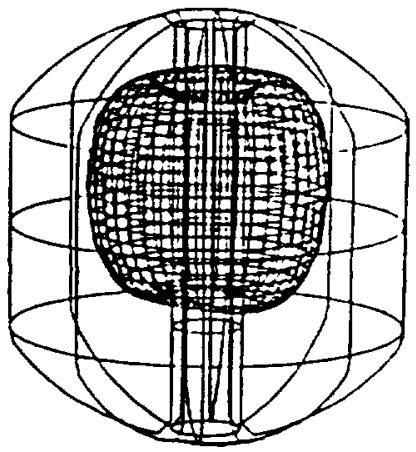

Fig. 7 
TIME

VARIATIONS OF

FLUID ANGULAR

MOMENTUMS

$\left(H_{x}, H_{y}, H_{z}\right)$

$\omega=0.1 \mathrm{rpm}$

$\tau=1200 \mathrm{~s}$

$f=0.1 \mathrm{~Hz}$
है

-

$=$

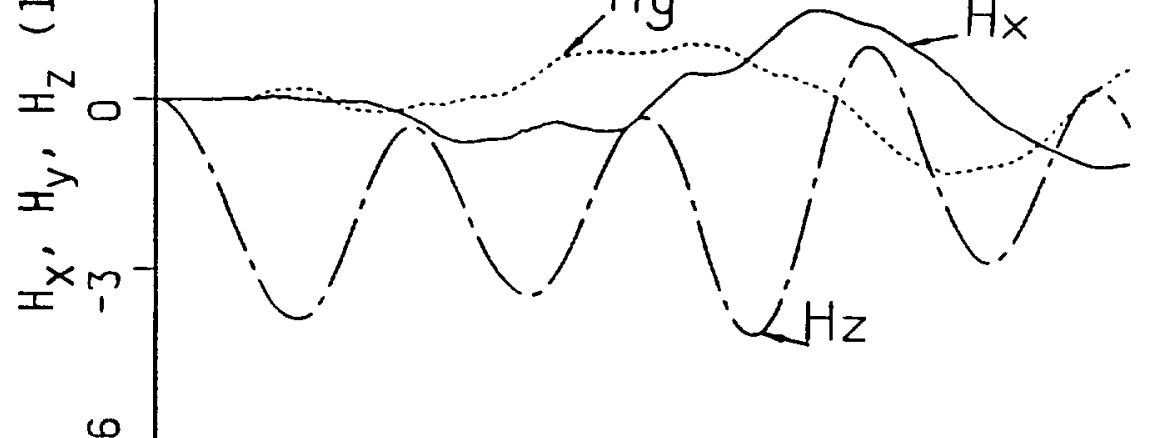

$\frac{\bar{s}}{\tilde{v}_{\mathrm{E}}}=7 \quad$ (B) $\mathrm{g}=10^{-7} \mathrm{~g}_{0}$

.

$=$

N
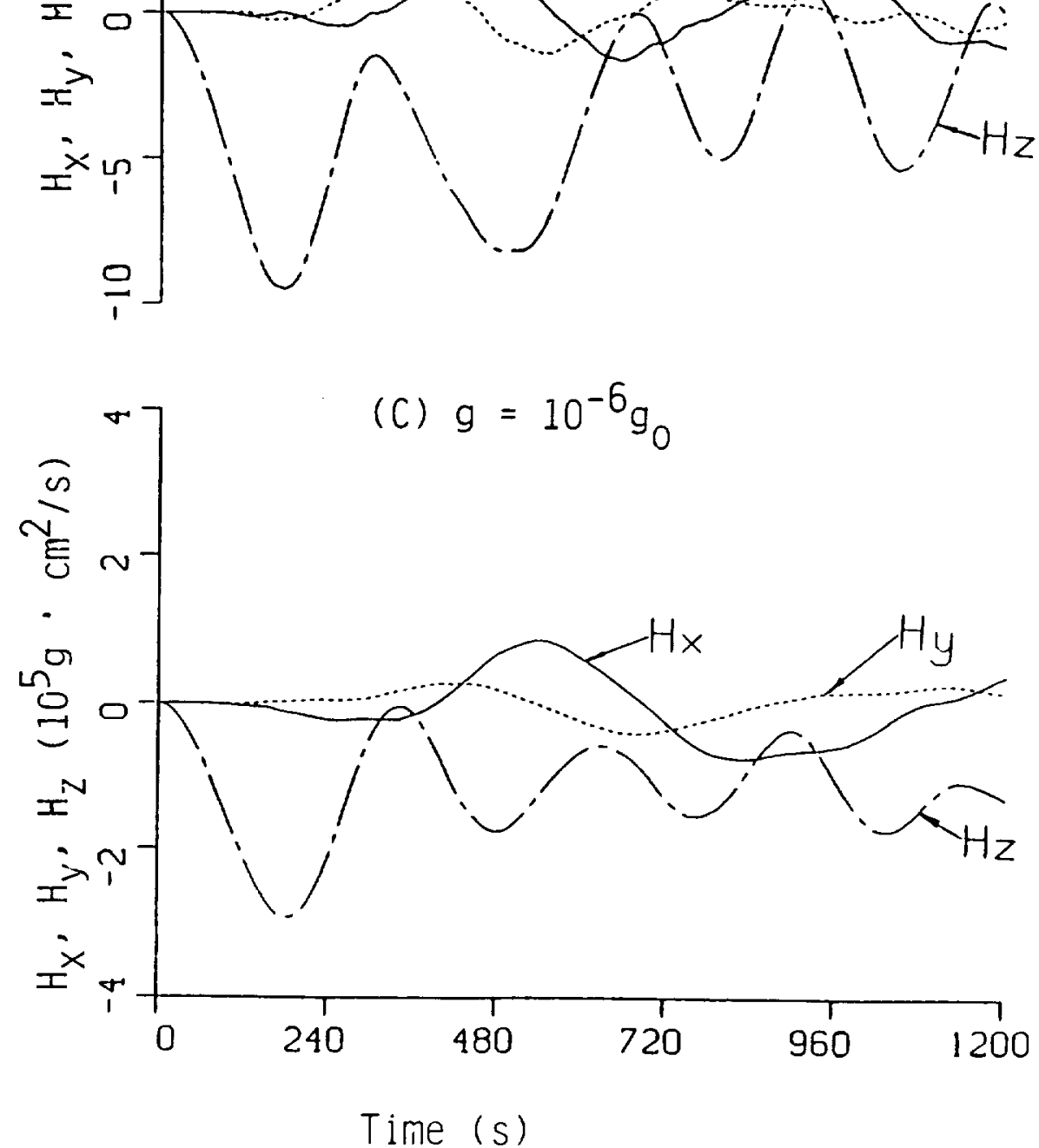

Time (s)

Fig 8 
TIME
VARIATIONS

OF FLUID

MOMENTS

$\left(M_{x}, M_{y}, M_{z}\right)$

$g=10^{-8} g_{0}$

$\omega=0.1 \mathrm{rpm}$

$\tau=1200 \mathrm{~s}$

$f=0.1 \mathrm{~Hz}$
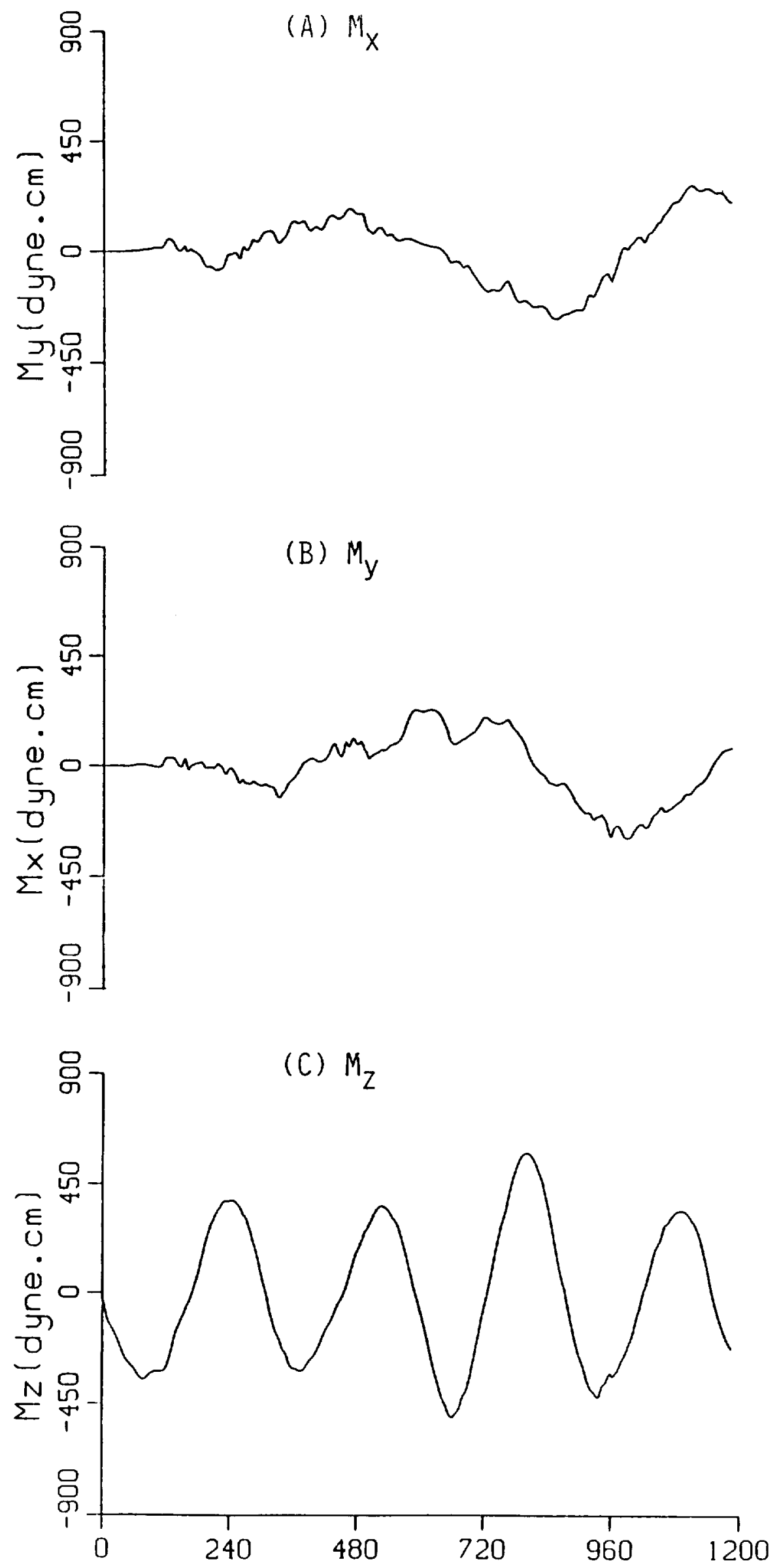

Time (s) 


\section{TIME \\ VARIATIONS \\ OF}

BUBBLE MASS

CENTER

$\left(X_{C}, Y_{C}, Z_{C}\right)$

$\omega=0.1 \mathrm{rpm}$

$\tau=1200 \mathrm{~s}$

$f=0.1 \mathrm{~Hz}$
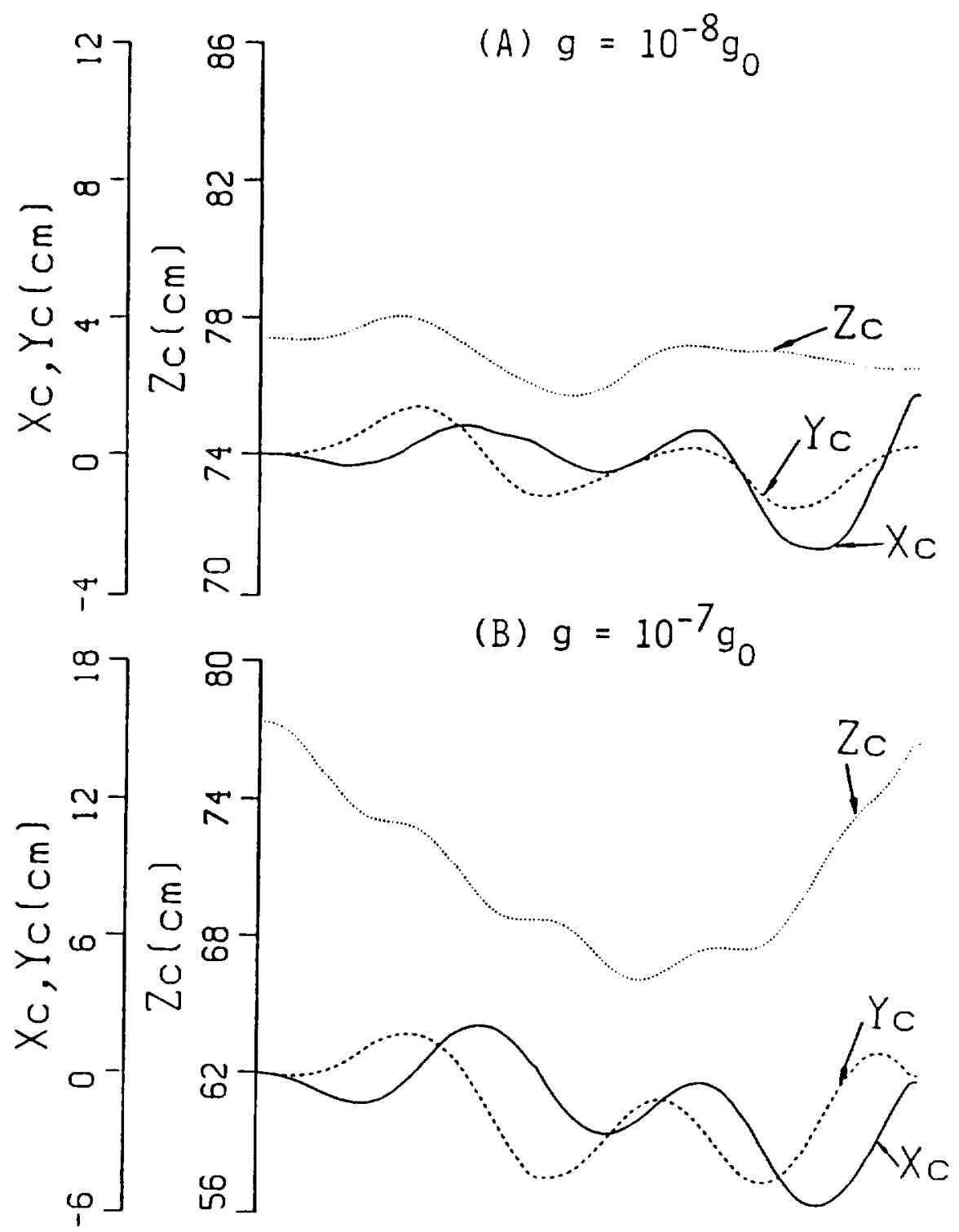

Fig. 10

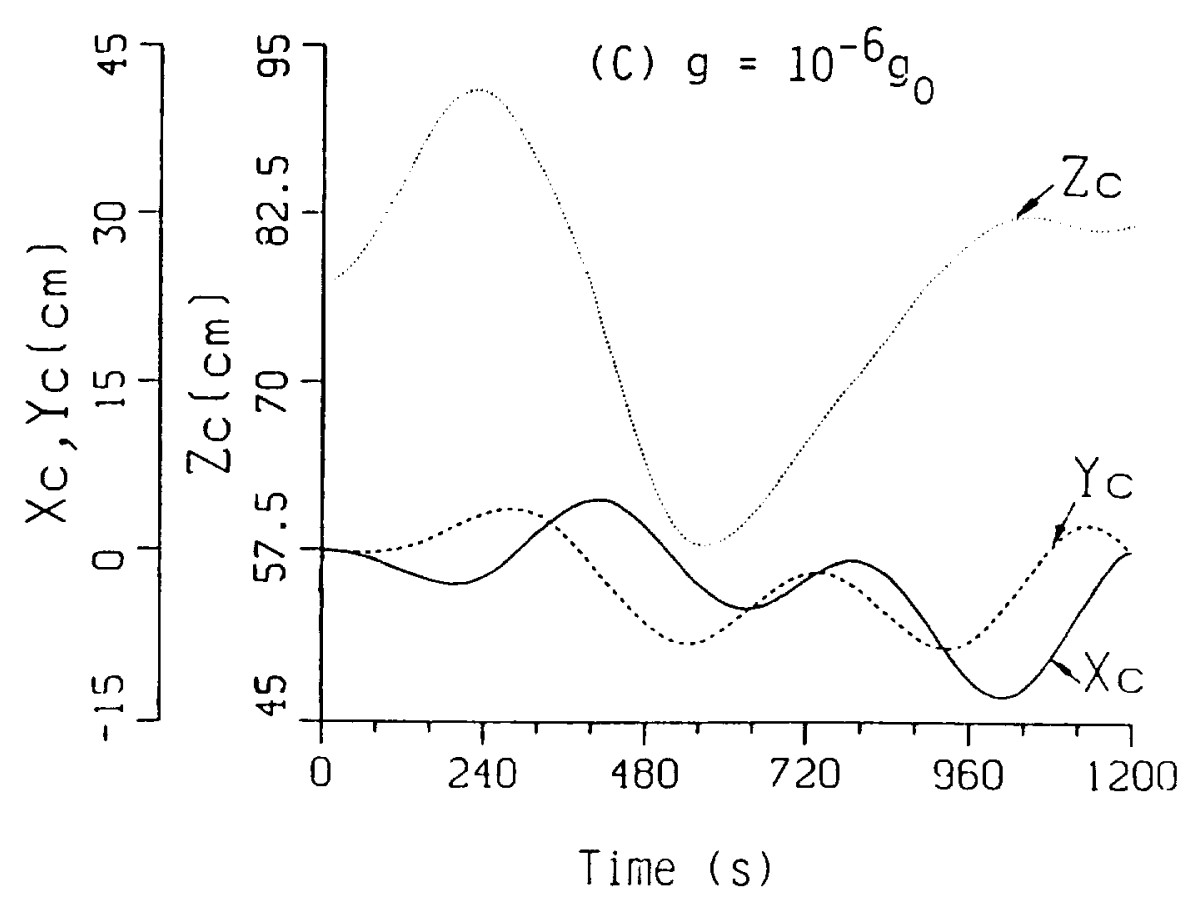




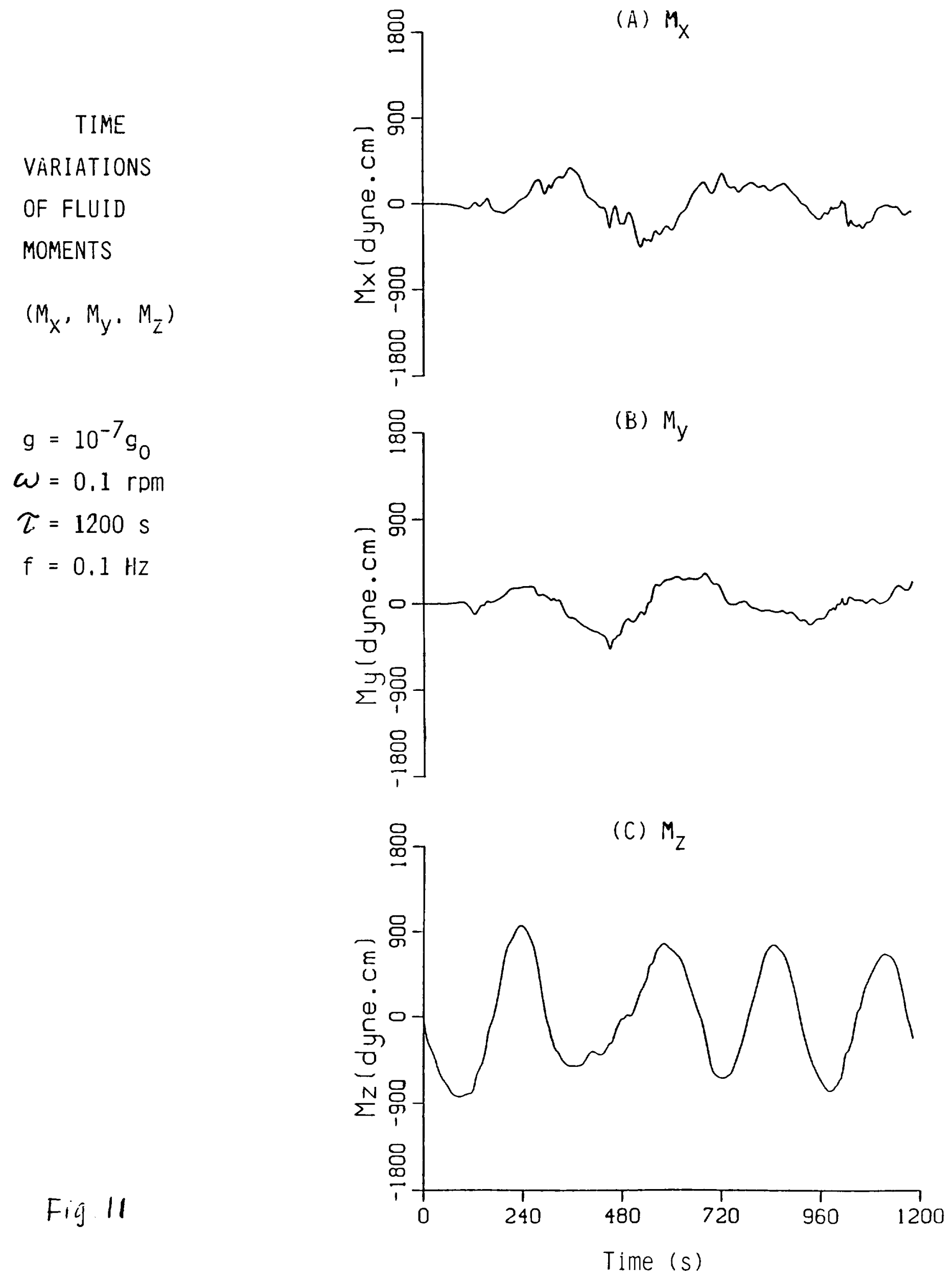




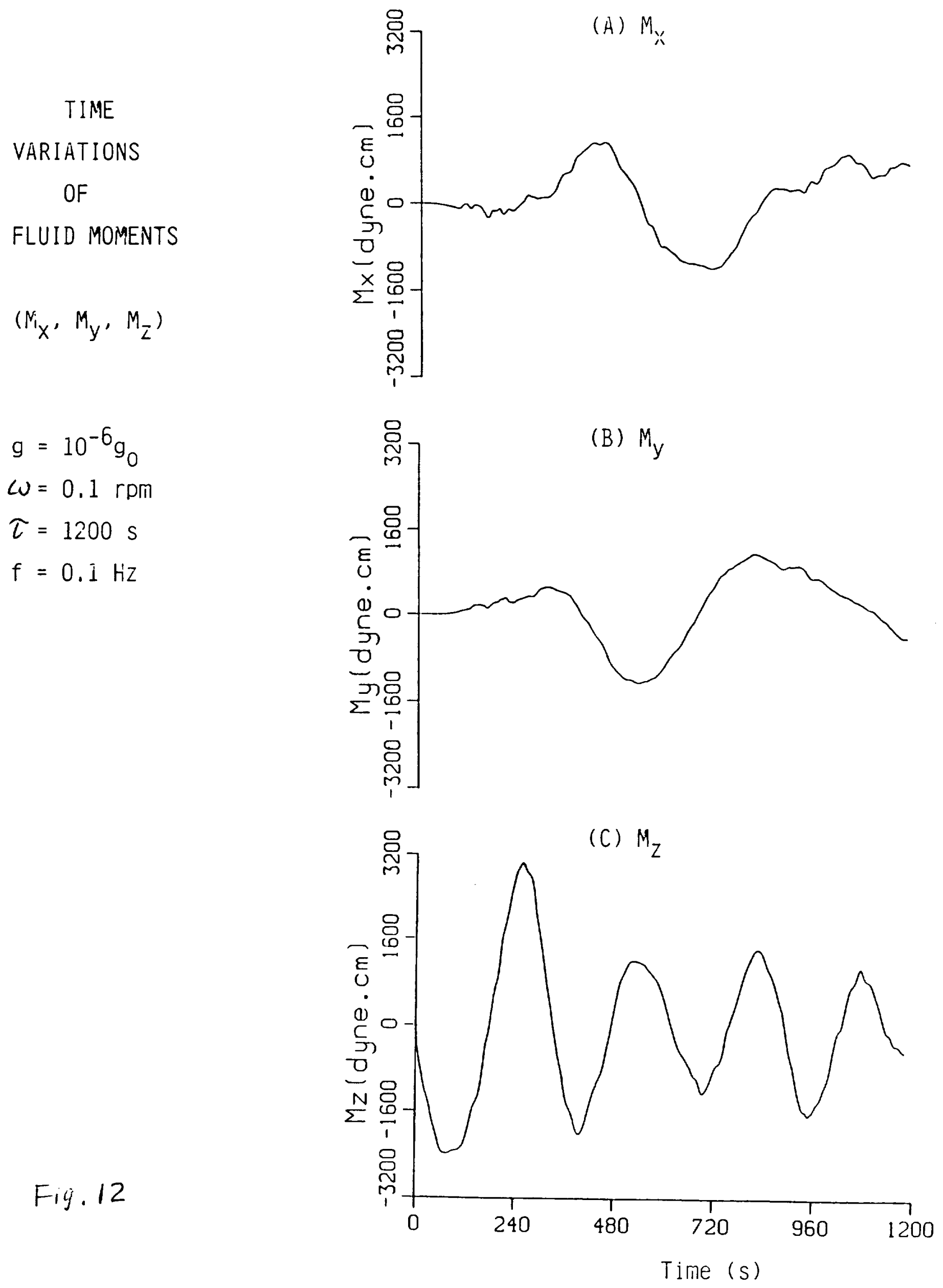

